\title{
New Formulation of Nonlinear Kinematic Hardening Model, Part II: Cyclic Hardening/Softening and Ratcheting
}

\author{
Volodymyr Okorokov ***a, Yevgen Gorash *, Donald Mackenzie* and Ralph van Rijswick** \\ " University of Strathclyde, 99 George St, Glasgow, G1 1RD, the UK \\ ***Weir Minerals, Venlo, 5928 PH
}

\begin{abstract}
The second part of the study presents development of the Dirac delta functions framework to modelling of cyclic hardening and softening of material during cyclic loading conditions for the investigated in Part I low carbon S355J2 steel. A new criterion of plastic strain range change is formulated. This provides more certainty in the cyclic plasticity modelling framework compared to classical plastic strain memorization modelling. Two hardening parameters from the developed kinematic hardening rule are written as functions of both plastic strain range and previously accumulated plastic strain. This representation of hardening parameters is able to accurately match experimental results with different types of loading programs including random loading conditions and considering initial monotonic behavior with yield plateau deformation. Ratcheting behaviour is simulated by the developed cyclic plasticity framework by considering an approximated form of the Dirac delta function for modelling the deviation effect and introducing an additional supersurface for better prediction of ratcheting rate. The proposed cyclic plasticity model requires up to 21 material constants, depending on application. A clear and straightforward calibration procedure, where sets of material constants are determined for each plasticity phenomenon considered, is presented. Application of the model to different materials under various tension-compression and non-proportional axial-torsion cycles shows very close agreement with test results.
\end{abstract}

Keywords: Cyclic plasticity, Cyclic hardening and softening, Strain range dependence, Ratcheting

volodymyr.okorokov@strath.ac.uk 


\section{Introduction}

A new formulation of nonlinear kinematic hardening model based on a Dirac delta function approach has been presented in a companion paper (Part I). The proposed model incorporates accurate description of stressstrain curve including transitions at load reversal, variation of Young's modulus after plastic deformation and the effect of the first stress invariant. Here the modelling framework is extended to include cyclic hardening/softening and ratcheting.

The phenomena of cyclic hardening and softening during cyclic plastic deformation are observed in many metals. Cyclic softening takes place in uniaxial tests with fixed strain range and zero mean strain where stress amplitude tends to stabilize under smaller stress values than from initial loading. Cyclic hardening, in turn, occurs as stabilization of stress under larger stress values.

From modelling perspective, cyclic hardening and softening effects can be represented in a cyclic plasticity modelling framework by incorporating the plastic strain range memory effect in the constitutive equations of isotropic and kinematic hardening rules. Initially, the effect of plastic strain range dependence was modelled through introduction of a plastic memory surface (Chaboche, 1979, McDowell, 1985a, b, Nouailhas et al., 1985, Ohno, 1982). The strain range dependence is incorporated into a variable of the isotropic hardening rule responsible for asymptotic stress saturation (Chaboche, 2008, Chaboche et al., 2013, Khutia et al., 2015, Taleb et al., 2014, Zhang and Jiang, 2008). This approach can accurately represent changes in hysteresis loop peak stresses during cyclic deformation but cannot fully model variation in the loop shape (Krishna et al., 2009, $\mathrm{Xu}$ et al., 2016). Results of tests with cyclic hysteresis curves plotted as yield surface size vs. number of cycles from Hassan and Kyriakides (1994a) discussed by (Krishna et al., 2009) and the experimental investigation of S355J2 steel in the companion to this paper (Part I) show no dependence of elastic domain size on plastic strain range under cyclic loading, indicating this modelling basis contradicts experimental observation. Hassan et al. (2008), Kang et al. (2003), Krishna et al. (2009), McDowell (1987), Xu et al. (2016), Zhou et al. (2018) proposed also including variation of hardening in the kinematic hardening rule. Xu et al. (2016) proposed the transformation zone concept to accurately describe both the change of stress amplitude and variation of hysteresis loop elastic domain. The evolution rules of isotropic and kinematic hardening are synchronized by introduction of a logistic function describing three stages of the cyclic softening process. This allowed the model to describe the transformation process of the stabilised hysteresis loop shape for BLY 160 steel during plastic accumulation with reasonable accuracy but showed some difference with experimental results for the initial monotonic stress-strain curve and transient behavior of the curve shapes during stabilization at fixed strain ranges. Zhou et al. (2018) proposed a multiplicative form of the kinematic hardening rule, where a coefficient is introduced to describe variation of the shape of hysteresis loops of 316 stainless steels with an assumption that all Armstrong and Frederick (A-F) back stress components from the Chaboche model are proportional, such that back stresses from different plastic strain ranges are linearly dependent. The kinematic hardening coefficient is presented as a function of accumulated plastic strain and plastic strain range, with a new calibration procedure proposed for its identification. This model has shown good prediction ability of peak stress variation as well as stress-strain curve shape of stabilised hysteresis loops for the low cycle fatigue region.

Ohno et al. (2018) proposed revisiting the concept of plastic memory surface (Chaboche, 1979), highlighting the uncertainty of the choice of the evolution parameters of the plastic strain surface when cases of variation in mean plastic strain due to pre-straining and ratcheting may arise. A resetting scheme is proposed for plastic memory surface, in which the strain surface is reset to a point every cycle, providing definite values for the evolution parameters. The introduced plastic strain range surface re-evolves every cycle regardless of the previous pre-straining and ratcheting effects, where current plastic strain range is defined by the size of the surface attained in a previous cycle. It is demonstrated that the resetting scheme correctly calculates plastic strain ranges under proportional loading and for loading with small degree of non- 
proportionality. The resetting scheme provides stepwise behaviour for the plastic strain range, which is not present in any other model. This can simplify the calibration procedure and numerical solution algorithms.

Prediction of the ratcheting behavior of metals, which comprises of accumulation of plastic strain under loading with fixed force amplitude and non-zero mean force, is an important requirement for cyclic plasticity modelling. The A-F type of kinematic hardening with Chaboche back stress decomposition used in many models naturally accounts for the phenomenon of ratcheting, although with a tendency to overestimate the ratcheting rate. For better agreement with experimental results, Chaboche (1991), Ohno and Wang (1993a, 1993b) proposed modifying the A-F hardening components, where the dynamic recovery terms are activated only when the back stresses approach certain values. The idea of modifying the dynamic recovery term in A$\mathrm{F}$ hardening components is widely used when particular loading programs with uniaxial or multiaxial responses are considered (Abdel-Karim, 2009, 2010, Abdel-Karim and Ohno, 2000, Bari and Hassan, 2000, Chen and Jiao, 2004, Guionnet, 1992, Van Do et al., 2015, Yu et al., 2012, Zhang and Xuan, 2017). A similar feature of these models is that the parameters used to control the ratcheting rate are included in the kinematic hardening rule governing the form of the stress-strain curve shape defining the plastic modulus (Bari and Hassan, 2001). Although the ratcheting parameters are usually not critical in curve shape modelling, its influence may restrict plasticity models from description of the curve shape with requirements of increased accuracy, especially for the transition behavior between the initial monotonic curve and subsequent curves up to a cyclic saturation point. Hassan and Kyriakides (1994a, 1994b) have shown that cyclic hardening and softening behaviours of two steels, CS1018 with cyclic softening and SS304 with cyclic hardening, significantly affect ratcheting rates. There is a significant difference between the ratcheting behavior of CS1018 under stabilized and non-stabilized states, such that the ratcheting rate of the virgin material is higher, leading to rapid failure after a certain accumulation of plastic strain. Therefore, consideration of the actual hardening/softening behavior of materials with transition between virgin and cyclically stabilized states is a critical factor in ratcheting simulation.

In this paper, the Dirac delta function approach developed in a companion paper (Part I) is extended to describe the change of cyclic hardening properties of materials with variation in the strain range of cyclic loading. A new criterion for the strain range change during cyclic loading is presented. It is proposed to consider the change of plastic strain range in a stepwise manner, where transition between strain ranges occurs when the critical distance in plastic strain space stops growing. This can be considered as generalization of the re-setting approach (Ohno et al., 2018). Two hardening parameters of the kinematic hardening rule are formulated as functions of plastic strain range and previously accumulated plastic strain. The definition of previously accumulated plastic strain follows the approach of Voyiadjis et al. (2012), with its mathematical form written in terms of the Dirac delta function approach. The presence of the Dirac delta function in the constitutive model of supersurfaces provides simulation of a closed hysteresis loop of a loading cycle. Modelling of the deviation effect during ratcheting and mean stress relaxation behaviours is achieved by considering an approximated form of the Dirac delta function. For better representation of experimental results, an additional supersurface associated with shifted supersurfaces is introduced. Its evolution rule is formulated with the use of the hardening rules, such that no additional material parameters are required.

\section{Definition of plastic strain range and previously accumulated plastic strain}

The modelling approach presented in the companion paper (Part I) has shown that that the shape of any stress-strain curve can be precisely described by the kinematic hardening rule in the form:

$$
\dot{X}_{i j}=A B C D \exp \left(-\frac{2}{3} \frac{\left(X_{k l}-O_{k l}\right) n_{k l}}{B}\right) \dot{\varepsilon}_{i j}^{p}
$$


Application of equation (1) to experimental results for S355J2 steel showed that the material parameters $A$ and $B$ are not constants but depend on the cyclic strain range and accumulated plastic strain. The dependence of hardening constants on accumulated plastic strain was not considered in the early studies of plastic strain range dependence (Chaboche, 1979, Nouailhas et al., 1985, Ohno, 1982), however its consideration in constitutive modelling provides better description of the stress-strain curve shape, as shown by Zhou et al. (2018). Here, the idea of assigning both plastic strain range and accumulated plastic strain to the hardening constants is further developed with an assumption that the hardening parameters are described as stepwise dependencies of the plastic strain range and accumulated plastic strain and hence can be formulated in terms of the Dirac delta function approach.

The hardening parameters $A$ and $B$ are defined in the form $A(\bar{q}, \bar{p})$ and $B(\bar{q}, \bar{p})$, where $\bar{q}$ and $\bar{p}$ are plastic strain range and previously accumulated plastic strain respectively. The main feature of $\bar{q}$ and $\bar{p}$ is that they change in a stepwise manner, providing stepwise dependence of the $A$ and $B$ parameters on accumulated plastic strain. This has been shown to give more certainty in modelling strain range dependence (Ohno et al., 2018). The stepwise change of previously accumulated plastic strain $\bar{p}$ has also shown improved description of the stress-strain curve shape (Voyiadjis et al., 2012). To provide stepwise dependencies of $\bar{q}$ and $\bar{p}$, the Dirac delta function approach described in Part I is applied in the following way:

$$
\begin{aligned}
& \dot{\bar{p}}=\delta\left(G^{p}\right) \frac{\dot{G}^{p}}{\dot{p}}[p-\underline{\bar{p}}] \dot{p} \\
& \dot{\bar{q}}=\delta\left(G^{p}\right) \frac{\dot{G}^{p}}{\dot{p}}\left[\frac{L^{p}}{2}-\underline{\underline{q}}\right] \dot{p}
\end{aligned}
$$

where $\underline{q}$ and $\underline{p}$ are the plastic strain amplitude and previously accumulated plastic strain respectively, defined at previous time $t$ with a time delay $\tau$ as $\underline{q}=\bar{q}(t-\tau)$ and $\underline{p}=\bar{p}(t-\tau)$. $G^{p}$ is the plastic strain range change condition defined as:

$$
G^{p}=\frac{\dot{L}^{p}}{\dot{p}}+\left|\frac{\dot{L}^{p}}{\dot{p}}\right|
$$

with $\frac{\dot{L}^{p}}{\dot{p}}=\frac{\partial L^{p}}{\partial \varepsilon_{i j}^{p}} \frac{\dot{\varepsilon}_{i j}^{p}}{\dot{p}}=\frac{3}{2} \frac{\varepsilon_{i j}^{p}-\bar{\varepsilon}_{i j}^{p}}{L^{p}} n_{i j}$.

$L^{p}$ is the shortest distance between points of current plastic strain and plastic strain accumulated before the change of the strain range and is defined as:

$$
L^{p}=\sqrt{\frac{2}{3}\left(\varepsilon_{i j}^{p}-\bar{\varepsilon}_{i j}^{p}\right)\left(\varepsilon_{i j}^{p}-\bar{\varepsilon}_{i j}^{p}\right)}
$$

where $\bar{\varepsilon}_{i j}^{p}$ is previously accumulated plastic strain tensor, defined as:

$$
\dot{\bar{\varepsilon}}_{i j}^{p}=\delta\left(G^{p}\right) \frac{\dot{G}^{p}}{\dot{p}}\left[\varepsilon_{i j}^{p}-\bar{\varepsilon}_{i j}^{p}\right] \dot{p}
$$

Condition (4) is similar in form to the condition of load reversal described in Part I, meaning that change in the strain range occurs when the shortest distance between points of current plastic strain and previously accumulated plastic strain stops growing. This provides an instant change of the variables by the Dirac delta 
function. The two conditions are essentially identical in the case of proportional loading, defining the strain range changes with reversal of loading path, but they can occur independently in a general case of complex non-proportional loading. This is a generalization of the concept of Ohno et al. (2018), where the strain range changes stepwise with the number of counted cycles. In the proposed new model, the strain range also changes stepwise according to the condition (4), but without the need for counting cycles.

\section{Development of evolution rules for hardening parameters}

To establish evolution laws for the kinematic hardening parameters $A$ and $B$, it is convenient to consider stress-strain curves from stabilised plasticity hysteresis loops. To determine the stabilized hysteresis loops, several tests with the loading program of cycling under fixed strain range with zero mean strain were performed. Alternatively, only one increasing level test can be used to investigate the shape of stress-strain curves in stabilized state. Figure 1 shows the results of the test in terms of stress-strain curves of the last stabilized cycle. Stabilization usually occurred after $\sim 15$ cycles, such that no change of the curve shape is visible. Low cycle fatigue softening after a larger number of cycles is not considered here.

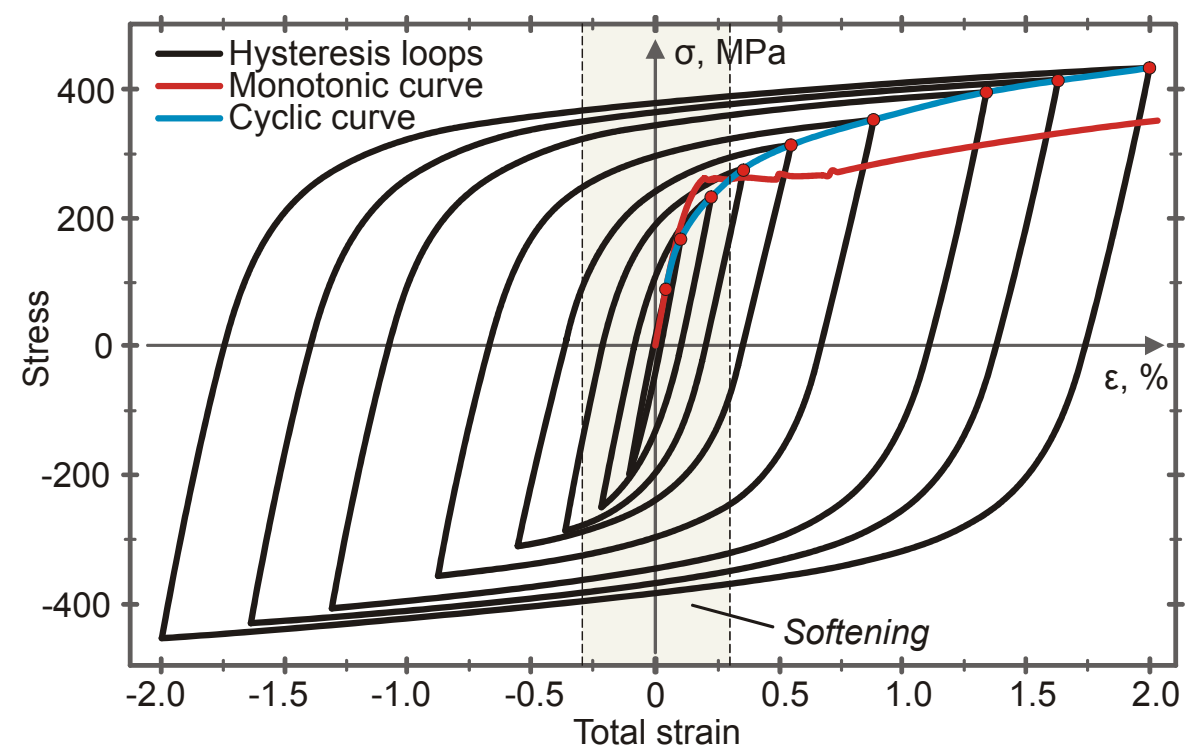

Fig. 1 - Stabilized hysteresis loops of S355J2 steel

As the material shows stabilization of the stress-strain curves with accumulation of plastic strain, it is convenient to express the parameters $A$ and $B$ through a saturation type of function in the form:

$$
\begin{aligned}
& \dot{A}=\mathrm{A}^{r}\left(\mathrm{~A}^{s}-A\right) \dot{\bar{p}} \\
& \dot{B}=\mathrm{B}^{r}\left(\mathrm{~B}^{s}-B\right) \dot{\bar{p}}
\end{aligned}
$$

where $\mathrm{A}^{s}$ and $\mathrm{B}^{s}$ are saturation values of $A$ and $B$ parameters and $\mathrm{A}^{r}$ and $\mathrm{B}^{r}$ are the rates of saturation.

All four parameters are functions of the plastic strain range, such that different saturation occurs under different strain ranges. The previously accumulated plastic strain $\dot{\bar{p}}$ and parameters $A$ and $B$ are variables with stepwise dependence on accumulated plastic strain. However, their mutual dependence is described by a smooth function with saturation effect after integration of equations (7) and (8). To determine parameters $\mathrm{A}^{s}$ and $\mathrm{B}^{s}$, the model is calibrated to the saturated stress-strain curves from stabilized hysteresis loops. Figure 2 shows an example application of the model to three strain ranges of the stabilized hysteresis loops, defined as mean curves (Section 4.2), and placed at the origin of the coordinate system relatively their reference points. It is shown that the three cases are very effectively described by the proposed model. 

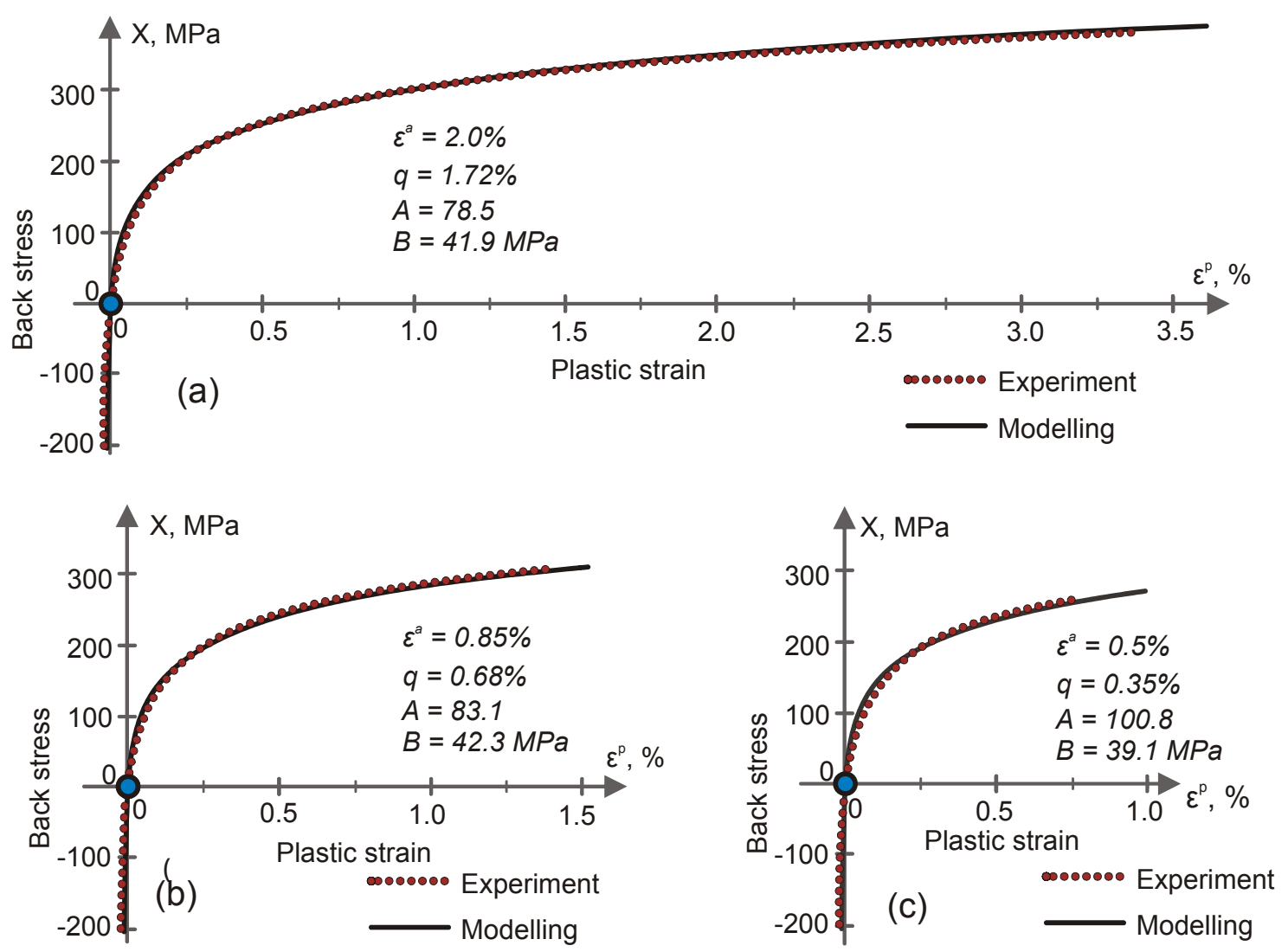

Fig. 2 - Stress-strain curves of stabilized hysteresis loops of S355J2 steel with modelling for strain amplitudes of (a) $2.0 \%$, (b) $0.85 \%$ and (c) $0.5 \%$

With calibration of more curves for different plastic strain ranges, calibrated $A$ and $B$ parameters can be plotted versus plastic strain amplitude as shown in Fig. 3. Both parameters have saturation values for plastic strain amplitudes above $\sim 1.4 \%$, which means the hardening process is stabilized for plastic amplitudes larger than this. As the $A$ and $B$ parameters are determined at their saturated state, parameters $\mathrm{A}^{s}$ and $\mathrm{B}^{s}$ can be defined by a saturation function in its incremental form:

$$
\begin{array}{r}
d \mathrm{~A}^{s}=\alpha^{r}\left(\alpha^{s}-\mathrm{A}^{s}\right) d \bar{q} \\
d \mathrm{~B}^{s}=\beta^{r}\left(\beta^{s}-\mathrm{B}^{s}\right) d \bar{q}
\end{array}
$$

where $\alpha^{r}, \alpha^{s}, \beta^{r}$ and $\beta^{s}$ are material constants, determined by fitting to the points from Fig. 3 with initial conditions $\left.\mathrm{A}^{s}\right|_{\bar{q}=0}=\mathrm{A}_{0}^{s}$ and $\left.\mathrm{B}^{s}\right|_{\bar{q}=0}=\mathrm{B}_{0}^{s}$.

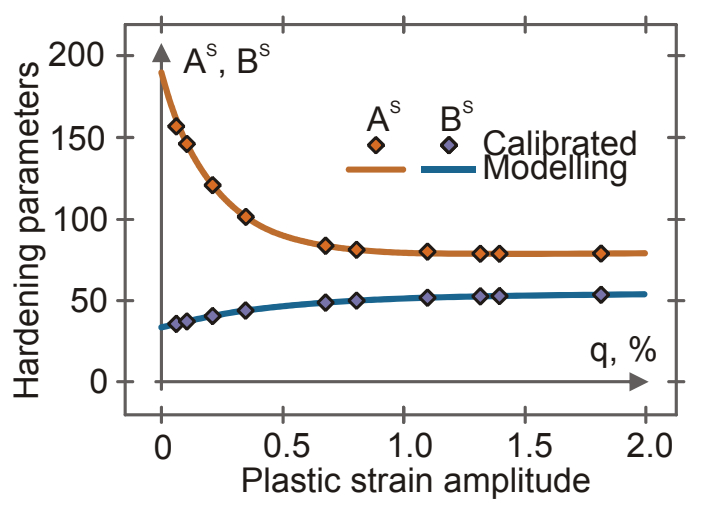

Fig. 3 - Strain range dependence of saturation values of hardening parameters at the stabilized state for S355J2 steel 
At the next stage, parameters $\mathrm{A}^{r}$ and $\mathrm{B}^{r}$, which are responsible for the rate of saturation of $A$ and $B$ with accumulation of plastic strain, are determined. For this, stress-strain curves from the saturation process under different plastic strain ranges are used. Figure 4 shows the deformation stabilization process under total strain amplitude of $1.3 \%$. Several curves extracted from this graph with their reference points placed at the origin of the coordinate system are shown in Fig. 5. Hardening parameters for the monotonic curve are found for the curves occurring after the plateau region. It is seen that all the curves from the saturation process are precisely modelled by the proposed kinematic hardening rule.

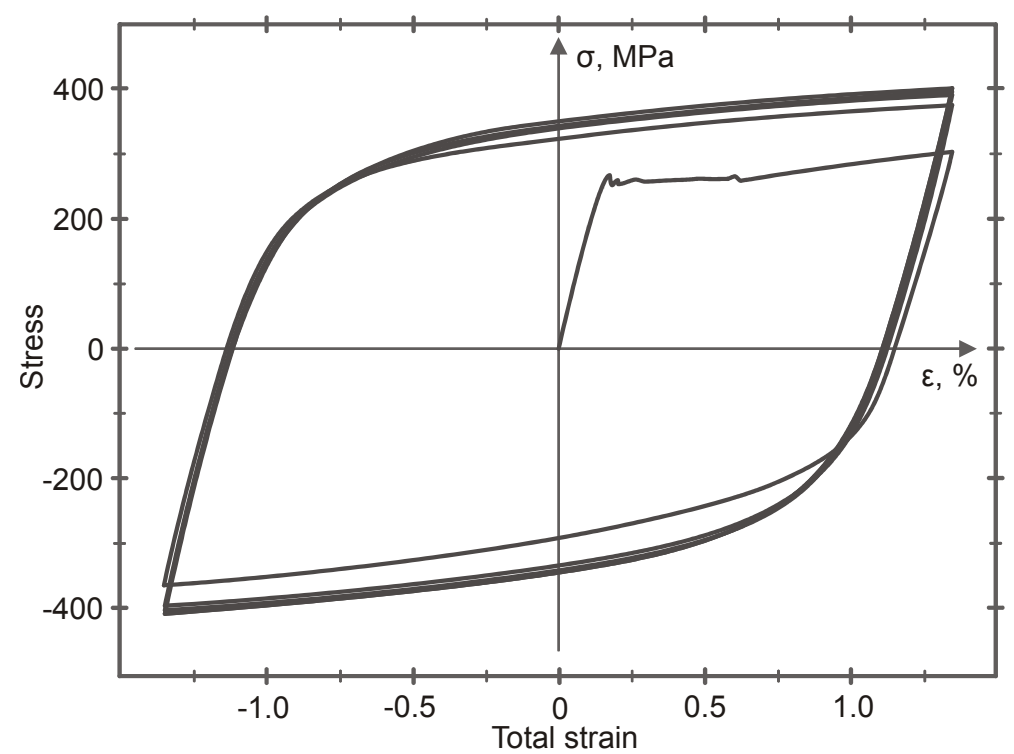

Fig. 4 - Stress-strain curve of loading at $1.3 \%$ of strain range for S355J2 steel

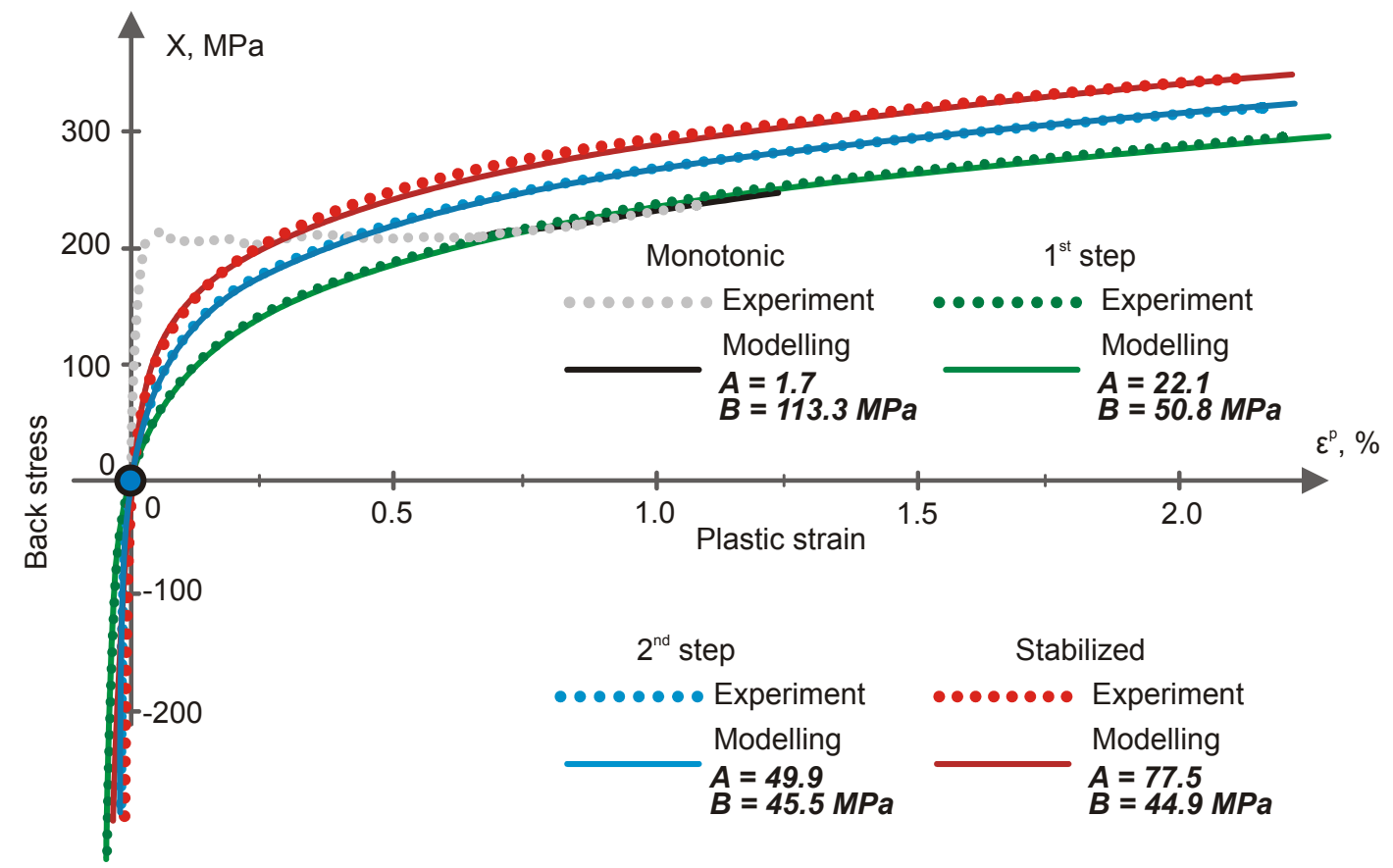

Fig. 5 - Stress-strain curves of loading at $1.3 \%$ of strain amplitude with modelling for S355J2 steel

Evolution of $A$ and $B$ parameters with accumulated plastic strain from stress-strain curves at different strain ranges can be plotted as shown in Fig. 6. Assuming that evolution of $A$ and $B$ parameters is described by equations (7) and (8), their dependence on previously accumulated plastic strain $\bar{p}$ is expressed by a smooth function with a saturation effect, whereas dependence of $A$ and $B$ on accumulated plastic strain $p$ 
is developed in a stepwise manner. Therefore, material parameters $\mathrm{A}^{r}$ and $\mathrm{B}^{r}$ from equations (7) and (8), which represent the saturation rate, can be directly evaluated from this dependence for different plastic strain ranges. Plotting this on one graph, as in Fig. 7, suggests that the saturation rate of S355J2 steel does not depend on the plastic strain range. Thus the $\mathrm{A}^{r}$ and $\mathrm{B}^{r}$ parameters are reduced to material constants. It should be noted that these parameters may show dependence on the plastic strain range for other materials and as such can be written as functions of plastic strain amplitude:

$$
\dot{\mathrm{A}}^{r}=\xi^{r}\left(\xi^{s}-\mathrm{A}^{r}\right) \dot{\bar{q}} \quad \dot{\mathrm{B}}^{r}=\vartheta^{r}\left(\vartheta^{s}-\mathrm{B}^{r}\right) \dot{\bar{q}}
$$

where $\xi^{r}, \xi^{s}, \vartheta^{r}$ and $\vartheta^{s}$ are material constants.

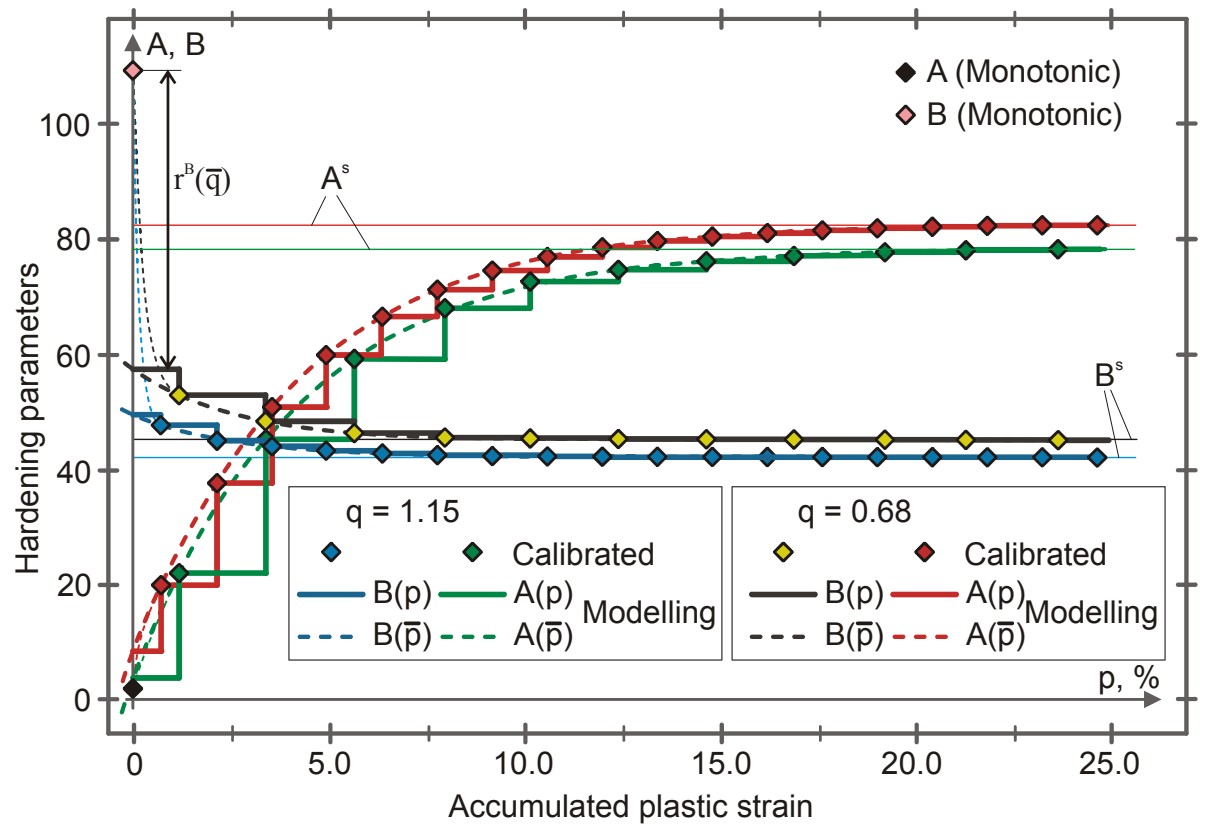

Fig. 6 - Evolution of hardening parameters with accumulation of plastic strain under loading at two strain ranges of $1.15 \%$ and $0.68 \%$ for $\mathrm{S} 355 \mathrm{~J} 2$ steel

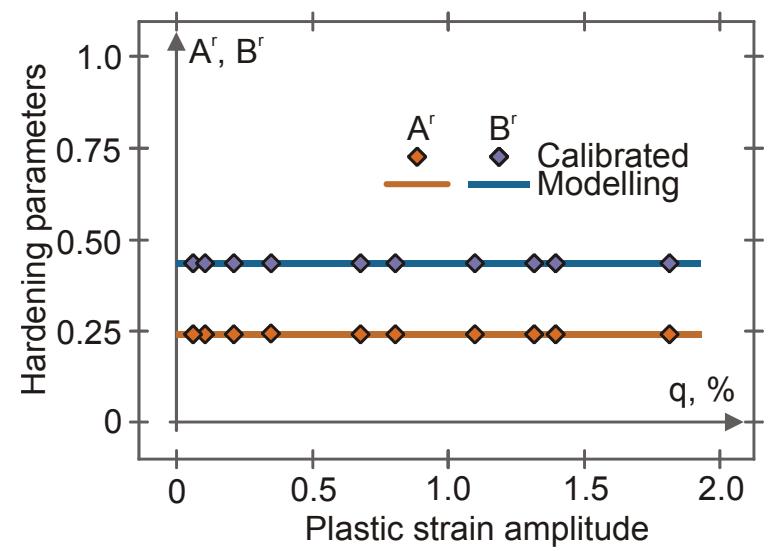

Fig. 7 - Strain range dependence of hardening parameters saturation rate for S355J2 steel

The present experimental investigation suggests that, compared to the monotonic stress-strain curve (Fig. 1), the material shows significant cyclic hardening under cyclic loading with strain amplitude greater than $0.29 \%$ and cyclic softening below this value. This behaviour is in accordance with experiments first performed by Landgraf et al. (1969) and is present in many other materials. There are limited approaches in the literature to model this behaviour. Krishna et al. (2009) introduced a threshold value for plastic strain amplitude $(0.29 \%$ for the investigated here $\mathrm{S} 355 \mathrm{~J} 2$ steel), below which an isotropic hardening parameter decreases and a 
kinematic hardening parameter increases, with an opposite behaviour for plastic amplitude above the threshold. In this way, cyclic softening is simulated for amplitudes below and hardening for amplitudes above the threshold. Essentially, this approach presents the isotropic and kinematic hardening parameters as functions of plastic strain amplitude. A similar approach to plastic strain range dependence is implemented in the model of the present study, where saturation values $\mathrm{A}^{s}$ and $\mathrm{B}^{s}$ and values of the rate of saturation $\mathrm{A}^{r}$ and $\mathrm{B}^{r}$ of hardening parameters $A$ and $B$ are dependent on plastic strain amplitude by equations (9), (10) and (11).

Experiments by Nouailhas et al. (1985) also suggest that cyclic hardening occurs with increasing strain range and show cyclic softening behaviour with decreasing strain range. From a modelling point of view, this behaviour can be taken into account by creating a nonhardening strain region (Ohno, 1982) or adding additional terms to plastic memory surface variables (Nouailhas et al., 1985) which allows the plastic memory surface to reduce under decreasing plastic strain range. In the current approach with Dirac delta function formulation for the strain range change and incorporation of the reference back stress tensor, there is no need for additional modifications to account for the phenomenon of cyclic softening with decreasing the strain range. Variation of the hardening parameters $A$ and $B$ are fully described by equations (7) and (8), where their values at a certain strain range are defined by the saturation values $\mathrm{A}^{s}$ and $\mathrm{B}^{s}$ and rate of saturation values $\mathrm{A}^{r}$ and $\mathrm{B}^{r}$, which are dependent on plastic strain range and previously accumulated plastic strain. This is seen for a particular case of strain amplitude decrease to $0.5 \%$ after stabilized cycling at strain amplitude of 2.0\% in Fig. 8, which shows the stress-strain curves from two stabilized hysteresis loops for this loading program. The hardening parameters from the stabilized hysteresis loop under $0.5 \%$ strain amplitude determined from the reference points are the same as from $0.5 \%$ strain range for loading with increasing strain and stress ranges.

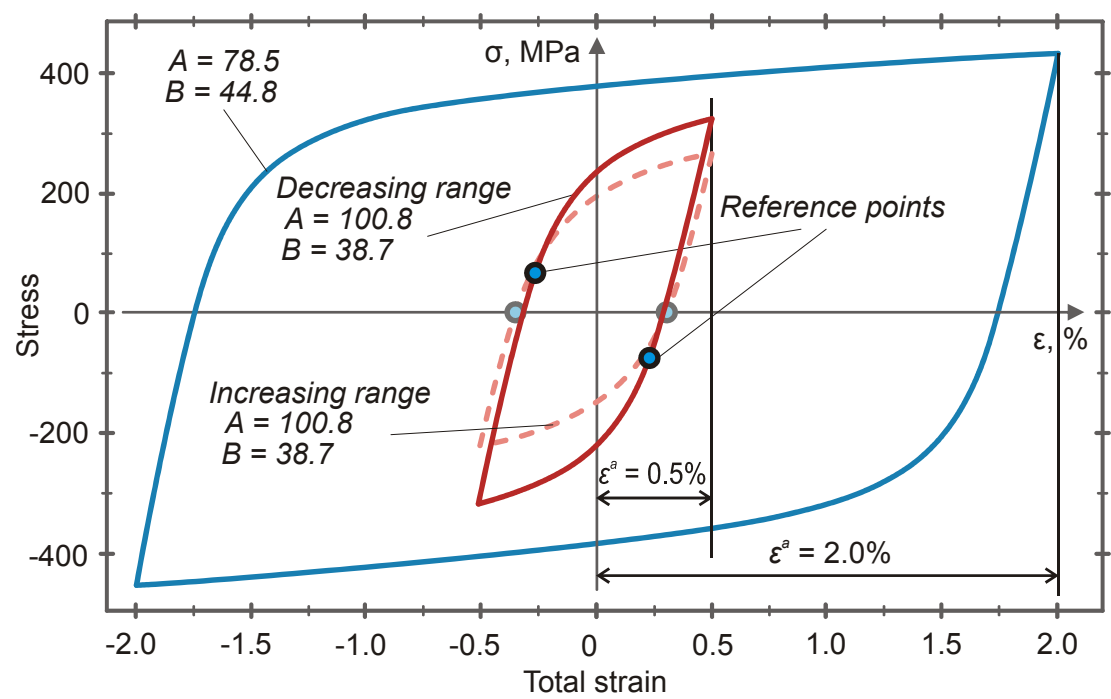

Fig. 8 - Comparison of two stabilized hysteresis loops under $1.0 \%$ of strain amplitude after different loading programs for $\mathrm{S} 355 \mathrm{~J} 2$ steel

The decreasing level curves look harder than curves from increasing loading. However, the actual hardening of the two cases is the same; it is the reference points of the stress-strain curves for decreasing loading are different. This shows that hardening parameters $\mathrm{A}^{s}, \mathrm{~B}^{s}, \mathrm{~A}^{r}$ and $\mathrm{B}^{r}$ are uniquely identified for this material by differential equations (7) and (8) regardless of loading conditions. Therefore, curves from Fig. 3 and Fig. 7, which show the dependence of these hardening parameters on plastic strain amplitude, are master curves for S355J2 steel. They establish the saturation values and rate of saturation of $A$ and $B$ parameters based on current plastic amplitude of deformation for any loading conditions. 


\section{Global and local hardening}

Variation of hardening parameters $A$ and $B$ in the form of equations (7) and (8) can precisely describe the cyclic hardening behaviour for loading conditions with increasing strain range amplitude together with increasing stress amplitude of every new loading cycle. In terms of stress supersurfaces $F_{0}^{s}, F_{1}^{s}$ and $F_{2}^{s}$ defined in the companion paper, Part I, these loading condition mean that loading reversal occurs when the current stress state is outside the shifted supersurfaces $F_{1}^{s}$ and $F_{2}^{s}$ defined by condition: $H(-\mu) \delta\left(G^{x}\right) \dot{G}^{x}$. $\mu$ defines a parameter which is equal to 1 inside the shifted supersurfaces and to 0 outside them:

$$
\dot{\mu}=\left(-h \sum_{i=0}^{2} \delta\left(F_{i}^{s}\right) \frac{\dot{F}_{i}^{s}}{\dot{p}}+\delta\left(G^{x}\right) \frac{\dot{G}^{x}}{\dot{p}} H(-\mu)\right) \dot{p}
$$

where parameter $h$ is defined as:

$$
h=\left[1-H\left(F_{0}^{r}\right)\right] H\left(-F_{1}^{r}\right) H\left(-F_{2}^{r}\right)
$$

It is convenient to denote the condition $\mu=0$ as global hardening. According to this, loading reversal occurs when the stress state is outside the shifted supersurfaces. Local hardening is then defined as $\mu=1$, when the stress state is inside the shifted supersurfaces. It will be shown that global and local hardening processes occur not only under a different reference point but also under different $A$ and $B$ parameters, even during the same loading step. To demonstrate this, several cases of loading at global and local states shown in Fig. 9 are considered. Figure 9a shows the stabilized state of global loading with hardening parameters equal to $A=78.3$ and $B=44.9$ at plastic strain amplitude of $2.0 \%$. If reversal of loading path occurs as shown in Fig. 9b, the strain amplitude will be confined within the value of $0.13 \%$ defining the local state of loading. According to the model of (7) and (8), this should lead to an immediate change of hardening parameters to $A=141.4$ and $B=38.2$, meaning cyclic softening. However, with return of the current stress from the local to global state at the stabilized curve, the hardening parameters are not significantly changed after local loading, which means that the plastic strain amplitude changing to a small value of local loading does not create the softening behaviour in a global sense. A similar situation is observed in Fig. 9c, where the global hardening parameters changed only to $A=80.2$ and $B=44.3$. More significant softening behaviour of global hardening occurs when the strain amplitude of the local state takes the values of $0.25 \%$ and $0.7 \%$, as shown in Fig. 9d and Fig. 9e. Subsequent increase in strain amplitude of the local state does not lead to a more pronounced softening behaviour at global loading, as suggested in Fig. 9f. This material behaviour is explained by an assumption that cycling at small plastic strain ranges of local loading with stresses inside supersurface, as from Fig. $9 \mathrm{~b}$ and Fig. 9c, is not able to significantly change the hardening behaviour at the global state.

Cycling at larger strain amplitudes of local loading, as shown in Fig. 9d and Fig. 9e, can change the global hardening to some extent. Further increase of the plastic strain amplitude of the local state does not lead to a change in hardening of the global behaviour, as the saturation values of $A$ and $B$ parameters at the stabilised global state are not very different from ones where cycling is confined within the supersurfaces defining the local hardening behaviour. One conclusion from this set of experiments is that the local hardening state occurs under hardening parameters different to the global state and it also affects hardening of the global loading state.

The test shown in Fig. 10 was performed to quantify the difference between the global and local hardening parameters $A$ and $B$ at cycling loading. The hardening parameters from the confined strain range of the local loading process can be determined as $A=147.4$ and $B=37.7 \mathrm{MPa}$. These hardening parameters have values 
which correspond to the saturated state with plastic strain amplitude of $q=0.13 \%$, as can be defined from Fig. 3. This means, full cyclic stabilization occurs whenever cyclic loading is fixed within a certain strain range. Once the stress state leaves local loading, crossing one of the supersurface $F_{2}^{s}$ in Fig. 10a or $F_{1}^{s}$ in Fig. $10 \mathrm{~b}$, the hardening parameters change partially towards values established at the stabilized state at $1.6 \%$ strain amplitude. It can also be concluded from Fig. 10a and Fig. 10b that crossing the supersurface at different points changes the hardening parameters to the same values. These values, in turn, depends on the stress range of cyclic loading of the local state. A larger stress range tends to change hardening parameters closer to values established at the stabilized strain amplitude of $1.6 \%$ of the global behaviour. To reflect these observation in constitutive modelling of hardening parameters, new variables $A^{g l}$ and $B^{g l}$ which are denoted as global hardening parameters are introduced.
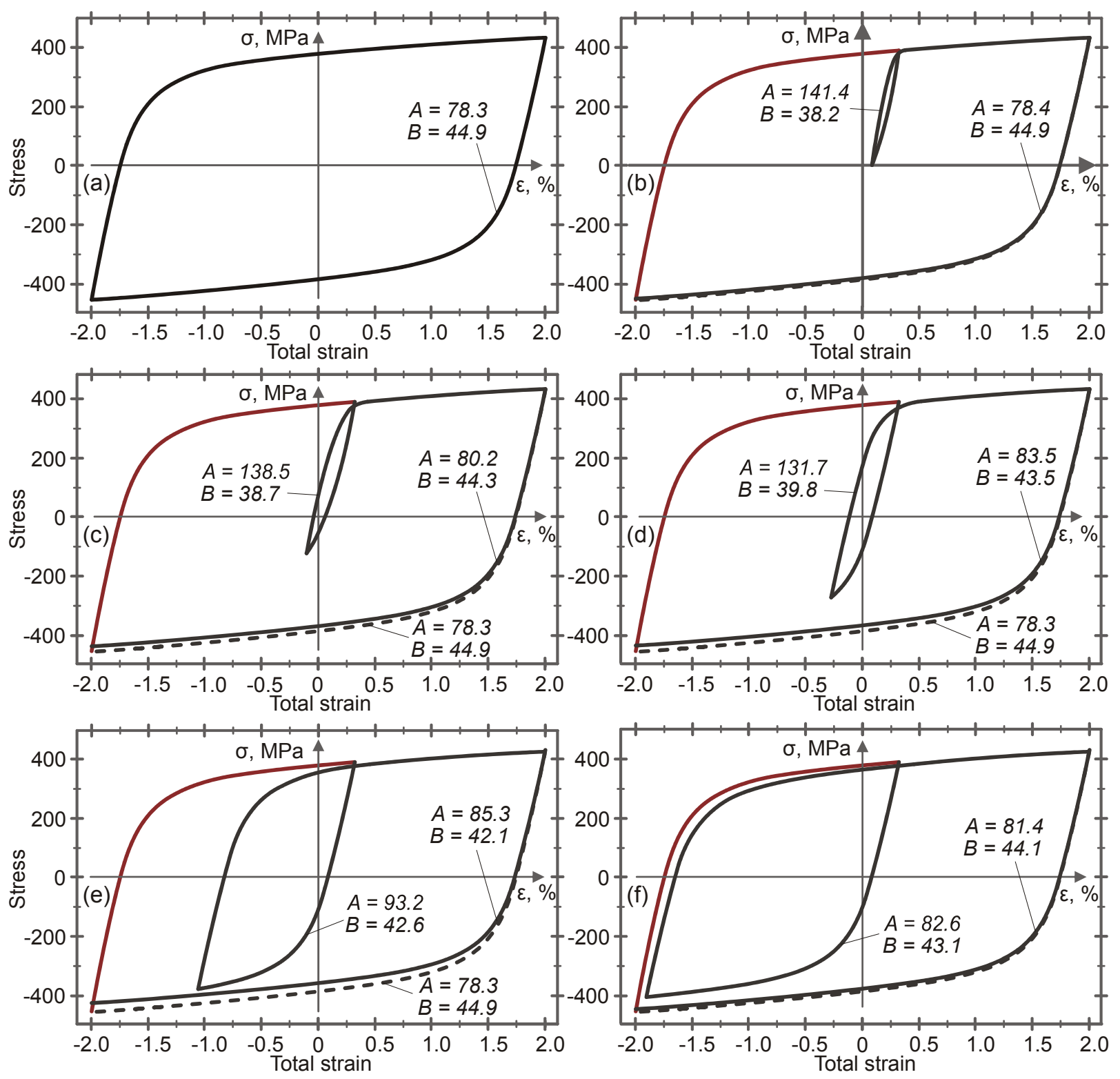

Fig. 9 - Effect of local deformation on global hardening of S355J2 steel; (a) stabilized hysteresis loop at 2.0\% of strain amplitude; change of global hardening after a local cycle with (b) $0.05 \%$, (c) $0.11 \%$, (d) $0.17 \%$, (e) $0.55 \%$ and (f) $0.97 \%$ of strain amplitude

To reproduce the change of hardening parameters $A$ and $B$ when the loading state crosses the boundary of one of the shifted supersurface, equations (7) and (8) are modified to the form: 


$$
\begin{aligned}
& \dot{A}=\mathrm{A}^{r}\left(\mathrm{~A}^{s}-A\right) \dot{\bar{p}}+\left[A^{g l}-\underline{A}\right]\left(1-\left(\frac{L^{g l}}{R^{s}}\right)^{\kappa}\right) h_{1} \dot{p} \\
& \dot{B}=\mathrm{B}^{r}\left(\mathrm{~B}^{s}-B\right) \dot{\bar{p}}+\left[B^{g l}-\underline{B}\right]\left(1-\left(\frac{L^{g l}}{R^{s}}\right)^{\kappa}\right) h_{1} \dot{p}
\end{aligned}
$$

where $h_{1}=\sum_{i=0}^{2} \delta\left(F_{i}^{s}\right) \frac{\dot{F}_{i}^{s}}{\dot{p}}$. This condition means that whenever the loading state leaves local loading, hardening parameters $A$ and $B$ change partially to global values $A^{g l}$ and $B^{g l}$. Partial change depends on the term $1-\left(L^{g l} / R^{s}\right)^{\kappa}$. Here, $\kappa$ is a material parameter and $L^{g l}$ is the global critical length in stress space between the current stress state and a stress state attained before loading reversal in global loading. It is not changed when loading reversals occur at the local state of loading. Thus, it is written in the following form:

$$
\dot{L}^{g l}=\delta\left(G^{x}\right) \frac{\dot{G}^{x}}{\dot{p}} H(-\mu)\left[L-L^{g l}\right] \dot{p}
$$
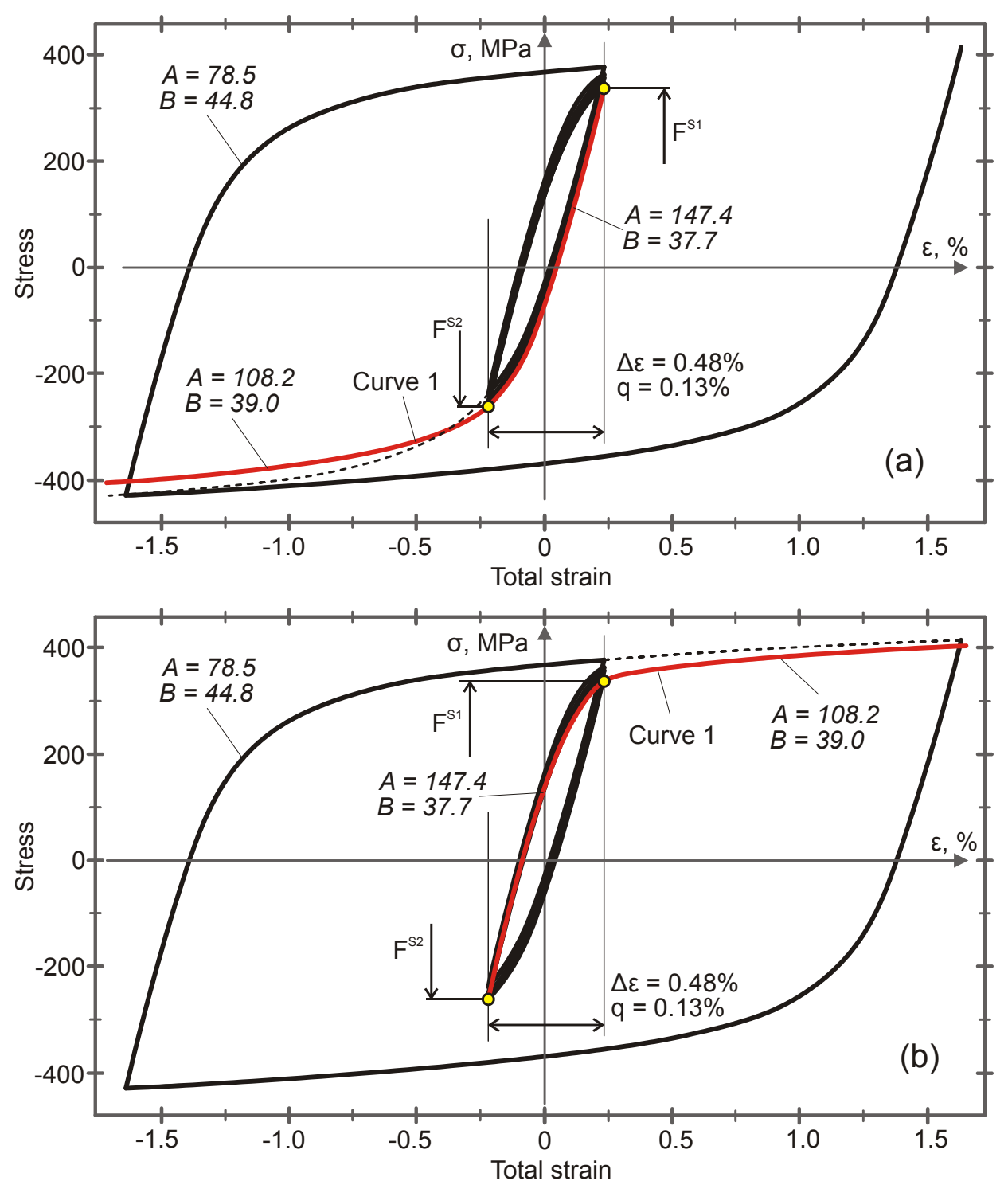

Fig. 10 - Effect of local deformation on global hardening of S355J2 steel 
According to (14) and (15), partial change to the global hardening parameters depends on the proportion of distance $L^{g l}$ to radius of the reference supersurface $R^{s}$, such that if this distance is small compared to the radius, current values of hardening parameters $A$ and $B$ almost fully turn into global parameters $A^{g l}$ and $B^{g l}$ as in the cases of Fig. $9 \mathrm{~b}$ and Fig. 9c. With increasing distance $L^{g l}$, global parameters $A^{g l}$ and $B^{g l}$ are influenced more significantly, as shown in Fig. 9d and Fig. 9e. When $L^{g l} \approx R^{s}$, as in the case of Fig. 9f, global parameters $A^{g l}$ and $B^{g l}$ take the values of parameters $A$ and $B$ almost without changing.

To provide that global parameters $A^{g l}$ and $B^{g l}$ are memorized during local loading, they are written in the following form:

$$
\begin{aligned}
& \dot{A}^{g l}=\delta\left(G^{x}\right) \frac{\dot{G}^{x}}{\dot{p}} H(-\mu)\left[\underline{A}-\underline{A}^{g l}\right] \dot{p} \\
& \dot{B}^{g l}=\delta\left(G^{x}\right) \frac{\dot{G}^{x}}{\dot{p}} H(-\mu)\left[\underline{B}-\underline{B}^{g l}\right] \dot{p}
\end{aligned}
$$

Equations (17) and (18) mean that if loading reversal occurs during global loading state, global hardening parameters $A^{g l}$ and $B^{g l}$ take current values of $A$ and $B$ corresponding to cycling loading in the global state. Otherwise, $A^{g l}$ and $B^{g l}$ remain unchanged.

To ensure correct evolution of plastic strain amplitude during loading reversals, a global variable for the plastic strain amplitude is introduced in a similar way to the $A^{g l}$ and $B^{g l}$ parameters as follows:

$$
\dot{\bar{q}}^{g l}=\delta\left(G^{p}\right) \frac{\dot{G}^{p}}{\dot{p}} H(-\mu)\left[\underline{\bar{q}}-\underline{\bar{q}}^{g l}\right] \dot{p}
$$

The evolution rule of plastic strain amplitude is then modified to:

$$
\dot{\bar{q}}=\delta\left(G^{p}\right) \frac{\dot{G}^{p}}{\dot{p}}\left[\frac{L^{p}}{2}-\bar{q}\right] \dot{p}+h_{1}\left[\bar{q}^{g l}-\bar{q}\right] \dot{p}
$$

Equation (20) provides that local cycling loading at small plastic strain range does not erase the memory of cycling loading at larger plastic strain range.

\section{Hardening of initial monotonic loading}

Examination of the results obtained in Fig. 6 suggests that evolution of hardening parameters $A$ and $B$ does not directly describe the transition part of the behaviour from the initial monotonic stress-strain curve. This happens because of an abrupt change of the hardening parameters during reversal of loading from the monotonic stress-strain curve. In this case, the functions integrated from the evolution equations (7) and (8) are not able to represent the transition behaviour. One way to fully describe this transition region is to develop another evolution rule of $A$ and $B$, such that its integration will give the correct transition behaviour. In order not to totally modify the developed functional dependence of the form of (7) and (8), which shows good agreement with experiments for all loading cases except for the transition effect of the monotonic stress-strain curve, it is proposed to include special transient functions $r^{A}(\bar{q})$ and $r^{B}(\bar{q})$ to return hardening parameters to the correct values when unloading from the monotonic stress-strain curve occurs. These functions are formulated with dependence on plastic strain amplitude in the form: 


$$
\begin{aligned}
& r^{A}(\bar{q})=\left(r_{1}^{A} \bar{q}+r_{2}^{A}-A_{0}^{g l}\right)\left(1-\left(\frac{L^{g l}}{R^{s}}\right)^{\kappa}\right) \\
& r^{B}(\bar{q})=\left(r_{1}^{B} \bar{q}+r_{2}^{B}-B_{0}^{g l}\right)\left(1-\left(\frac{L^{g l}}{R^{s}}\right)^{\kappa}\right)
\end{aligned}
$$

where $r_{1}^{A}, r_{2}^{A}, r_{1}^{B}$ and $r_{1}^{B}$ are material constants and $A_{0}^{g l}$ and $B_{0}^{g l}$ are initial values of global hardening parameters corresponding to the hardening of the monotonic stress-strain curve. Functions $r^{A}(\bar{q})$ and $r^{B}(\bar{q})$ are essentially the difference between the values of hardening parameters predicted by equations (7) and (8) and actual determined values. To provide an additional instant change of hardening parameters at loading reversal from the initial monotonic stress-strain curve together with subsequent reversals, equations (14) and (15) are modified to the form:

$$
\begin{aligned}
& \dot{A}=\mathrm{A}^{r}\left(\mathrm{~A}^{s}-A\right) \dot{\bar{p}}+\left[A^{g l}-\underline{A}-r^{A}(\bar{q})\right]\left(1-\left(\frac{L^{g l}}{R^{s}}\right)^{\kappa}\right) h_{1} \dot{p}+\delta\left(G^{x}\right) \frac{\dot{G}^{x}}{\dot{p}} H(-\mu) r^{A}(\bar{q}) \dot{p} \\
& \dot{B}=\mathrm{B}^{r}\left(\mathrm{~B}^{s}-B\right) \dot{\bar{p}}+\left[B^{g l}-\underline{B}-r^{B}(\bar{q})\right]\left(1-\left(\frac{L^{g l}}{R^{s}}\right)^{\kappa}\right) h_{1} \dot{p}+\delta\left(G^{x}\right) \frac{\dot{G}^{x}}{\dot{p}} H(-\mu) r^{B}(\bar{q}) \dot{p}
\end{aligned}
$$

Equations (23) and (24) ensure that whenever loading reversal occurs during the monotonic behaviour, hardening parameters $A$ and $B$ obtain additional values by an instant change of $r^{A}(\bar{q})$ and $r^{B}(\bar{q})$ to conform to the experimental results. The transient functions $r^{A}(\bar{q})$ and $r^{B}(\bar{q})$ adjust the transition behaviour depending on the proportion of distance $L^{g l}$ to radius $R^{s}$, which provides the different effect of these functions after local cycling at small plastic strain ranges. The functions stop affecting the model behaviour when $L^{g l} / R^{s}=1$.

\section{Ratcheting prediction}

\subsection{Delta function framework for ratcheting simulations}

Ratcheting behaviour of material occurs as a progressive accumulation of plastic strain under fixed loading force with a non-zero mean force value. In the uniaxial case of loading, the ratcheting phenomenon occur as a deviation of stresses when they approach the upper or lower points of the hysteresis loop, creating additional accumulated strain $\Delta \varepsilon^{r}$ under constant force amplitude as shown in Fig. 11. On crossing the value of stress from the upper point, the deformation process continues with global hardening parameters $A^{g l}$ and $B^{g l}$ under the reference point from the global deformation process. Approaching the stress boundary gradually decrease the reference point. Generalization of this behaviour to the multiaxial case can be done by introduction of two ratcheting surfaces (Fig. 12), written in the form:

$$
\begin{aligned}
& F_{1}^{r}=\sqrt{\frac{3}{2}\left(X_{i j}-\bar{\rho}_{i j}^{1}\right)\left(X_{i j}-\bar{\rho}_{i j}^{1}\right)}-R^{s} \\
& F_{2}^{r}=\sqrt{\frac{3}{2}\left(X_{i j}-\bar{\rho}_{i j}^{2}\right)\left(X_{i j}-\bar{\rho}_{i j}^{2}\right)}-R^{s}
\end{aligned}
$$


The back stress reference tensor is relaxed when the current stress state approaches the boundary of one of the ratcheting supersurfaces. To include the case of ratcheting supersurfaces, the shift of back stress reference tensor is updated by considering the ratcheting supersurface in the parameter $\mu$ instead of the shifted ones. The deviation parameter $\mu$, which is responsible for the transition between deformation curves and relaxation of back stress reference tensor, is modified to:

$$
\dot{\mu}=\left(-h \sum_{i=0}^{2} \phi\left(F_{i}^{r}\right) \frac{\dot{F}_{i}^{r}}{\dot{p}}+\delta\left(G^{x}\right) \frac{\dot{G}^{x}}{\dot{p}} H(-\mu)\right) \dot{p}, \quad \dot{F}_{0}^{r}=\dot{F}_{0}^{s}
$$

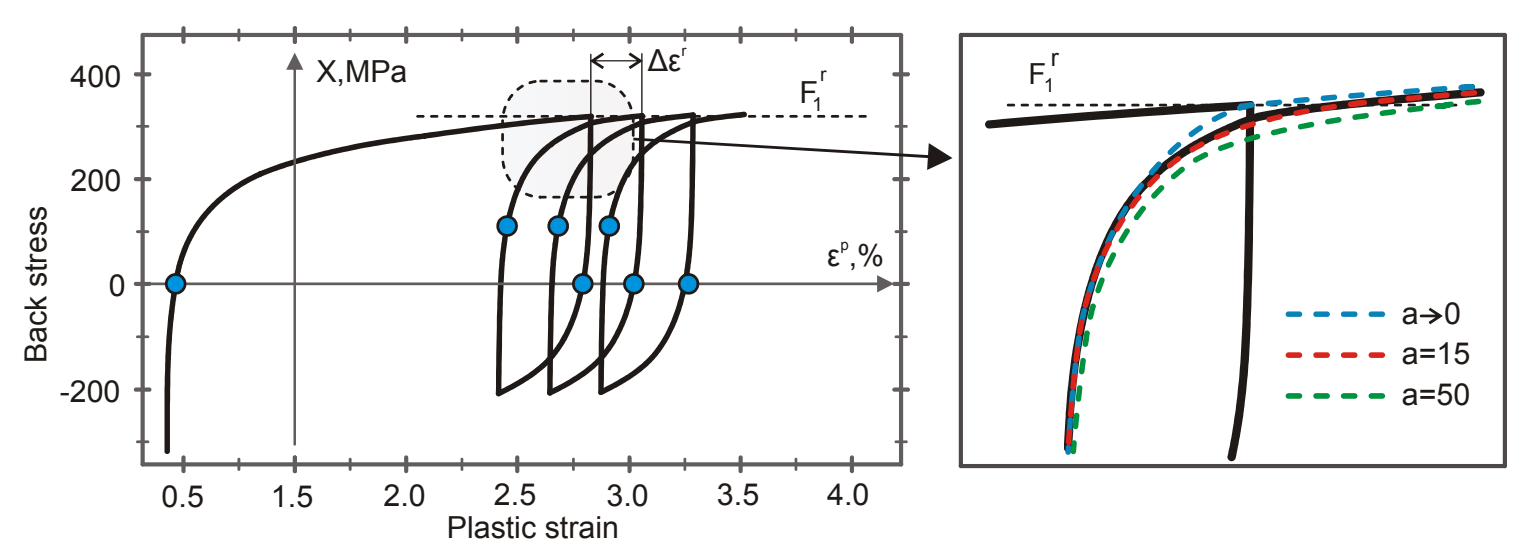

Fig. 11 - Ratcheting phenomenon of S355J2 steel with modelling

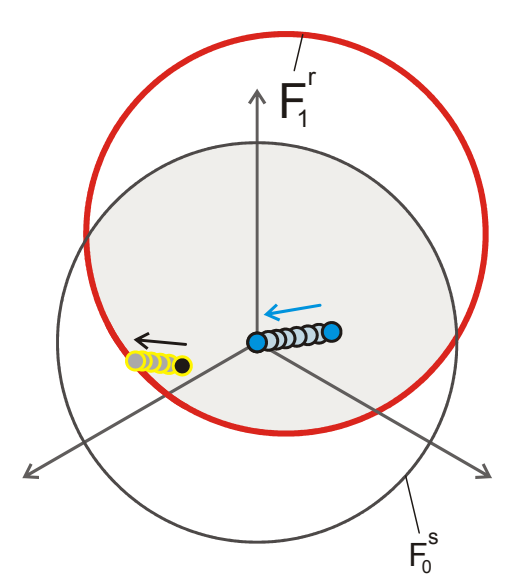

Loading before crossing the boundary

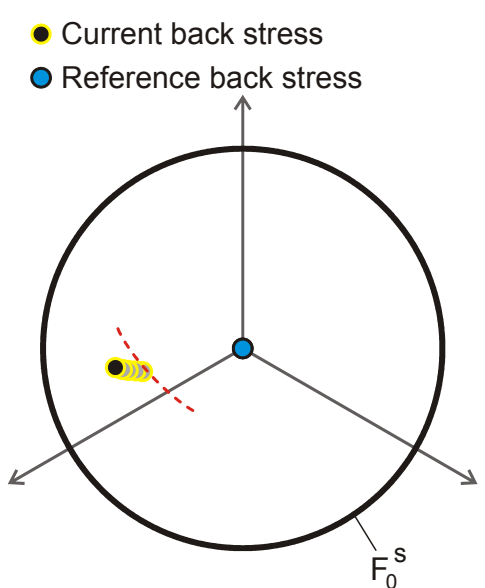

Loading after crossing the boundary

Fig. 12 - Multiaxial representation of the deviation effect modelling

Function $\phi$ is an approximate form of the Dirac delta function:

$$
\phi(x, a)=\exp \left(-\frac{x^{2}}{a^{2}}\right) / a \sqrt{\pi}
$$

where the Dirac delta function is expressed as $\delta(x)=\lim _{a \rightarrow 0} \phi(x, a)$.

Parameter $\mu$ is equal to one when the loading is inside the ratcheting supersurfaces and gradually takes zero when the current stress approaches the boundary of one of the supersurfaces, as shown in Fig. 12. Once one of the supersurfaces is crossed, the deformation process continues with the stress state inside or on the reference supersurface further evolving the reference supersurface. The back stress reference tensor is formulated as defined in Part I of the study: 


$$
O_{i j}=\frac{2 X_{y}-X_{h}}{2 X_{y}} \mu\left(Z_{i j}-\bar{X}_{i j}\right)
$$

Solution of equation (27) for uniaxial loading, illustrated in Fig. 11, shows that variation of the parameter $a$ of the function $\phi$ can model the deviation effect to different extents. It is noted that when $a \rightarrow 0$, there is an instant relaxation of the reference point, such that the loop is closed without the deviation effect. When the parameter $a$ takes finite values the deviation effect is simulated, giving a constant value of ratcheting strain extension $\Delta \varepsilon^{r}$. Therefore, as a default, the model simulates a constant rate of ratcheting. Terms $\dot{F}_{0}^{r} / \dot{p}, \dot{F}_{1}^{r} / \dot{p}$ and $\dot{F}_{2}^{r} / \dot{p}$ provide that the deviation effect occurs within a certain distance to the ratcheting supersurface boundary. The distance is the same regardless of type of loading and deformation history. The difference in the ratcheting rate for different types of loading is conditioned by the difference in stress-strain curve slope (plastic modulus) $\dot{\sigma} / \dot{\varepsilon}^{p}$ when the stress state approaches the boundary of the ratcheting supersurface. This follows the idea of uncoupled models (Bari and Hassan, 2001), where plastic modulus is not defined by kinematic hardening rules directly, as in the Dafalias and Popov (1975) model. This has shown better ratcheting behaviour description than in coupled types of models Chaboche (1991), Ohno and Wang (1993a).

By representing plastic deformation with the use of the developed framework of ratcheting supersurfaces, ratcheting can be considered as local cycling inside the reference supersurface $F_{0}^{s}$, where every new reversal of loading instantly moves the shifted supersurfaces $F_{1}^{s}$ and $F_{2}^{s}$. Loading inside a shifted supersurface defines the position of back stress reference tensor $O_{i j}$. Approaching of the boundary of a ratcheting supersurface, $F_{1}^{r}$ and $F_{2}^{r}$ move the back stress reference tensor to the zero position. The proposed translation of the ratcheting supersurfaces can also simulate cyclic mean stress relaxation and shakedown effects during ratcheting. The shakedown state occurs when the distance between a stress value and the boundary of the supersurface becomes large enough to cause the deviation effect, such that a cyclic hysteresis loop closes up.

\subsection{Cyclic hardening and softening in ratcheting behavior}

The phenomena of ratcheting and mean stress relaxation are usually affected by hardening and softening behaviours of material, especially at non-stabilized state (Hassan and Kyriakides, 1994a). Figures 13 and 14 show examples of ratcheting response of S355J2 steel under the two conditions. The first is stabilized cyclic loading under strain amplitude of $1.3 \%$. In the second condition, the ratcheting test was performed for an initial virgin condition of material. It is observed that for the case of stabilised cycling conditions, progressive ratcheting causes cyclic softening of the material which is observed as widening of the stress-strain hysteresis loop, as shown in Fig. 13b. At the same time, ratcheting of the non-stabilized case is accompanied by the cyclic hardening phenomenon, where the stress-strain hysteresis loop gets thinner with accumulation of plastic strain (Fig. 14b). Simulation of the stress-strain curve shape of the hysteresis loops from these cycling conditions demonstrates that the proposed model very accurately describe the curve shape under cyclic hardening and softening behaviour during ratcheting loading.

Both cases of stabilized and non-stabilized material show initial decelerating rate of ratcheting with stabilizing at constant ratcheting rate, as suggested in Fig. 13c and Fig. 14c. It is clear that in the case of ratcheting at the non-stabilized state, initial deceleration of the ratcheting rate is explained by progressive hardening of the material. Softening of the stabilized material should accelerate the ratcheting rate, however, experimental observation shows the opposite behaviour. This can be explained by weakening of the deviation effect with accumulation of ratcheting strain. From a modelling point of view, Hassan et al. (1992), Hassan and Kyriakides (1992, 1994a, 1994b) have shown that this behaviour is simulated by allowing bounds from Dafalias and Popov (1975) model to move in the direction of ratcheting. In the present model, this behaviour 
is modelled by translation of the ratcheting supersurfaces with accumulation of ratcheting strain. Translation of the centre of the ratcheting supersurfaces is developed as follows:

$$
\bar{\rho}_{i j}^{l}=\dot{\bar{c}}_{i j}^{l}+\delta\left(G^{x}\right) \frac{\dot{G}^{x}}{\dot{p}}\left[H\left(-F_{l}^{s}\right) r^{h}+H\left(-F_{l}^{s}\right)\left(\underline{\rho}_{-i j}^{l}-{\underline{c_{i j}}}_{l}^{l}\right)\right] \dot{p}, l=1,2
$$

where $r^{h}$ is a ratcheting parameter which can be taken in an associated form with hardening slope (plastic modulus) of deformation at global straining:

$$
\dot{r}^{h}=A^{g l} B^{g l} \exp \left(-\frac{X_{e q}}{B^{g l}}\right) H\left(L^{p}-\bar{q}\right) H\left(-F_{g l}^{s}\right) \dot{L}^{p}
$$

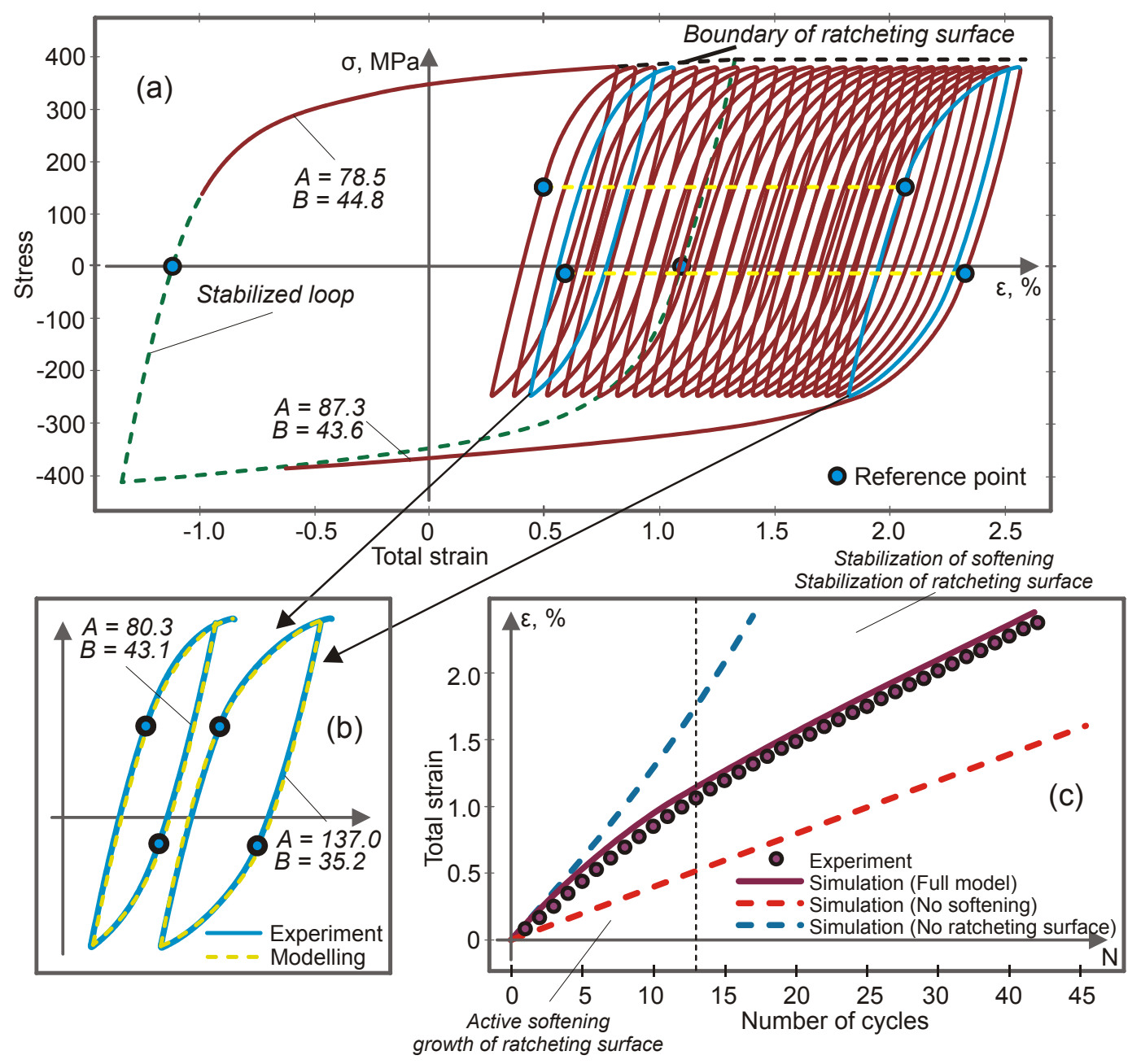

Fig. 13 - (a) Ratcheting of S355J2 steel from stabilized hysteresis loop; (b) two hysteresis loops from ratcheting deformation with modelling; (c) prediction of ratcheting rate

Equation (30) provides that the ratcheting supersurfaces $F_{1}^{r}$ and $F_{2}^{r}$ can translate towards the centre of the reference supersurface $F_{0}^{s}$. After reaching the centre of the reference supersurface, the ratcheting supersurface remains unchanged, providing a constant rate of ratcheting. Figs $13 \mathrm{c}$ and $14 \mathrm{c}$ show the results of simulation of ratcheting rate with the use of equation (30). In the case of the stabilized material from Fig. 13, the first 12 cycles show a competition between acceleration of ratcheting rate by the softening behaviour and its deceleration by movement of the ratcheting surface resulting in total deceleration by the dominance of the latter phenomenon. After stabilization of the softening and ratcheting supersurface translation, the ratcheting straining continues with a constant rate. In the case of the non-stabilized material with ratcheting starting from 
the initial monotonic stress-strain curve, the ratcheting supersurface does not evolve, as the peak stress of loading corresponds to the boundary of the reference supersurface. The deceleration of the ratcheting rate is simulated by initial hardening of the material following stabilization under constant rate after around 15 cycles of loading. Application of the developed model to the two cases of ratcheting shows that if the cyclic softening and hardening effects are not taken into consideration, the model shows a steady ratcheting rate and overestimates experimental results for both stabilized and non-stabilized conditions. Figs 15 and 16 show ratcheting simulations for the two cases of stabilized and non-stabilized materials under different loading conditions, where the tests are performed with constant maximum stress varying stress ratio defined as $R=\sigma_{\max } / \sigma_{\min }$. For the simulations, the deviation parameter $a$ from the approximated Dirac function (28) is calibrated to the ratcheting curve for the stabilized material at $R=-0.66$. It can be seen that all other curves are simulated with reasonable accuracy.

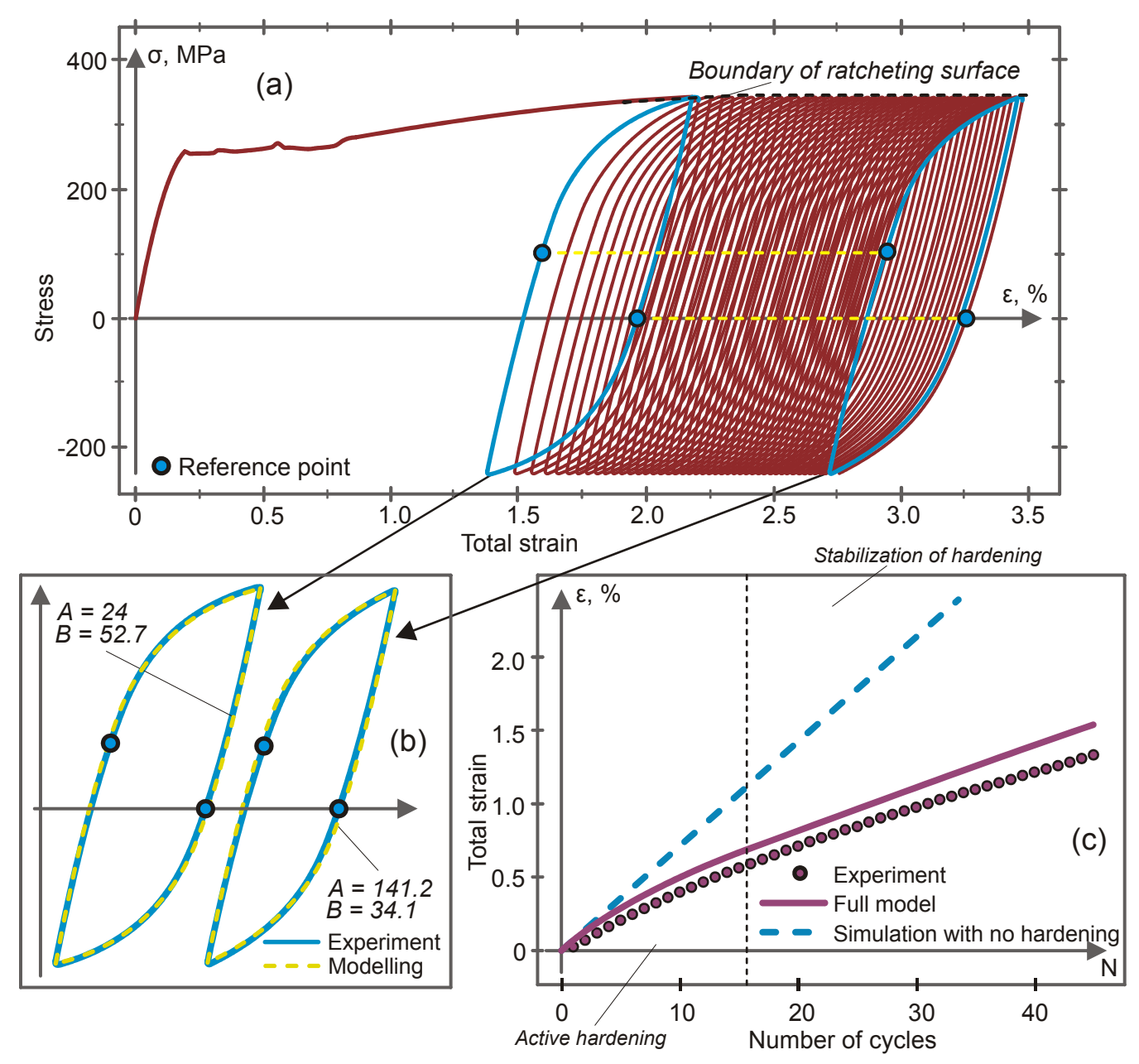

Fig. 14 - (a) Ratcheting of S355J2 steel from monotonic stress-strain curve; (b) two hysteresis loops from ratcheting deformation with modelling; (c) prediction of ratcheting rate 


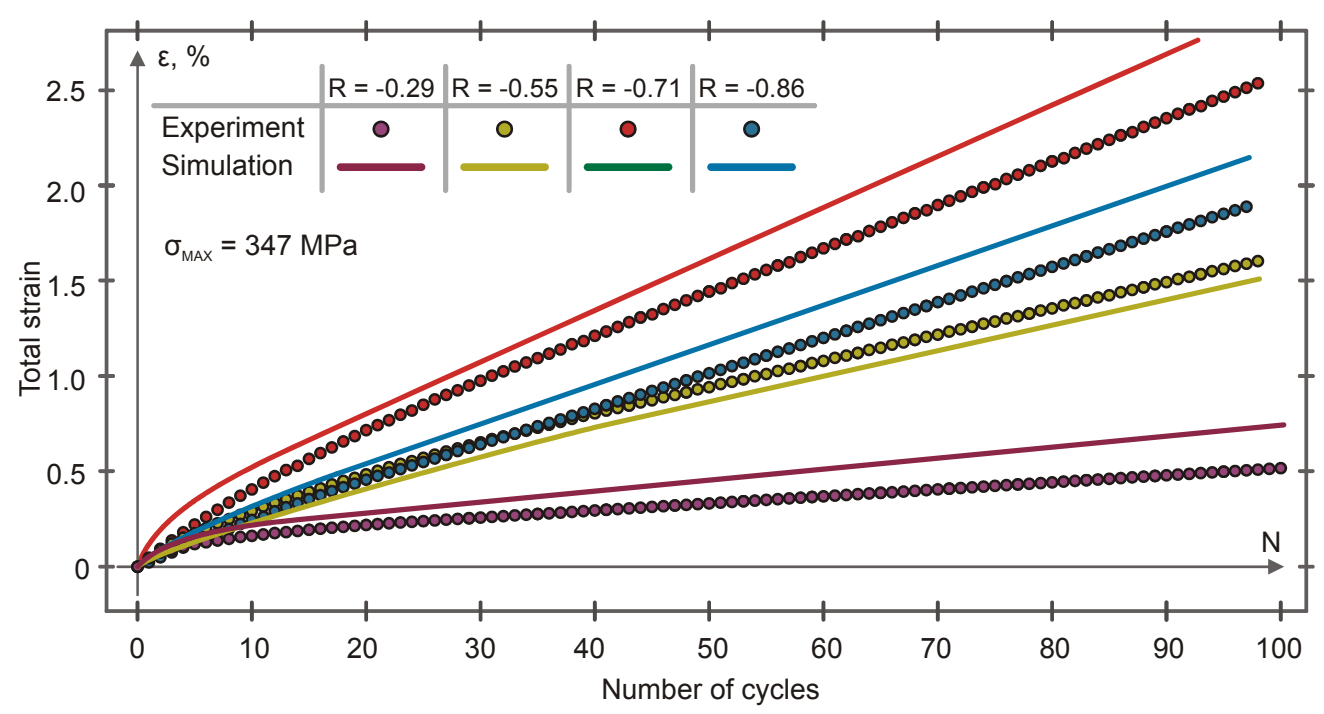

Fig. 15 - Prediction of ratcheting rate of S355J2 steel monotonic stress-strain curve

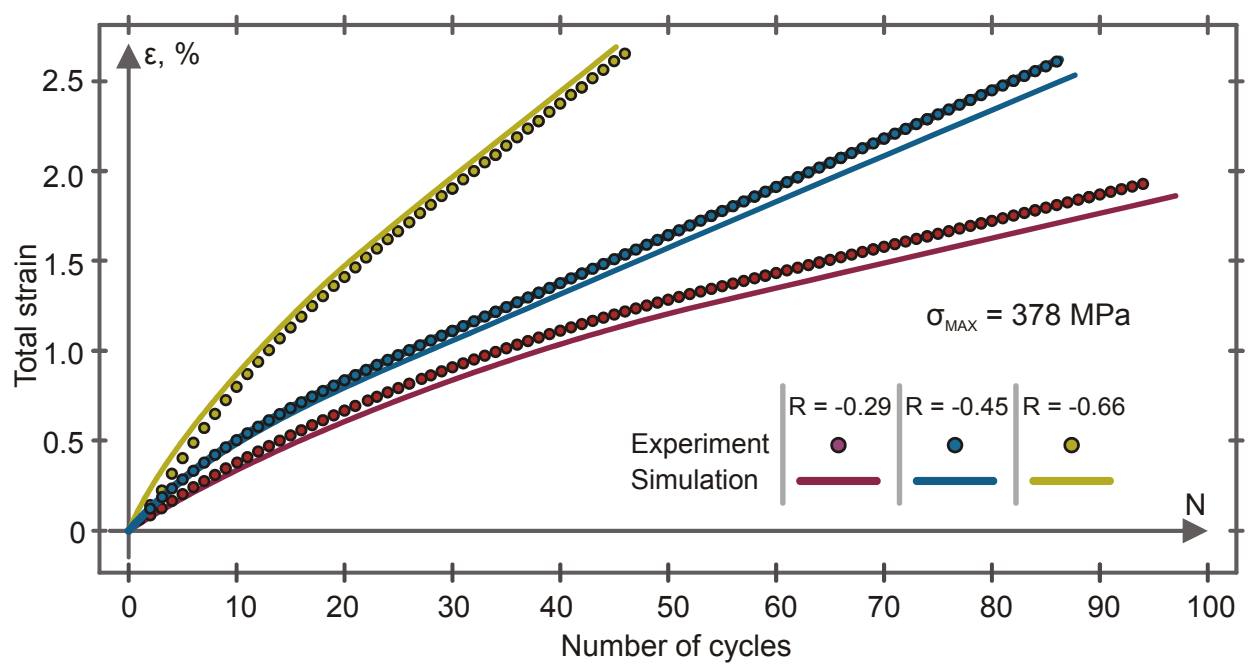

Fig. 16 - Prediction of ratcheting rate of S355J2 steel from stabilized hysteresis loop

\section{Identification of material parameters}

A summary of the developed constitutive model of cyclic plasticity is given in Table 1. Determination of numerical values for the model parameters is an important step in cyclic plasticity modelling. The procedure for material constant identification should be clear and straightforward, with the use of a minimum number of experimental test sets where, preferably, each set of parameters should be determined for each cyclic plasticity phenomenon considered independently. In the present investigation of the cyclic plasticity behavior of S355J2 low carbon steel, many experimental data sets are analysed to determine evolution rules for model variables. However, the material parameters identification procedure proposed here requires much less experimental data with a direct sequence of parameters determination per considered phenomenon.

\subsection{Isotropic hardening}

In many studies, the isotropic hardening variables are determined based on peak stresses variation with accumulation of plastic strain under different cyclic loading strain ranges. In this approach, the relation of isotropic hardening variable and the elastic domain is not consistent, as shown in the companion paper, Part I. In this study, the experimental data sets of yield stress identified by $0.01 \%$ of offset strain, presented in Part 
I, determined variation of the elastic domain with accumulation of plastic strain. It was established that the measured elastic domain drops initially with no visible change during subsequent plastic deformation. Therefore, the saturation type of isotropic hardening $\dot{R}=b(Q-R) \dot{p}$ is a good choice for describing this type of behavior, where parameters $Q$ and $b$ are directly evaluated from the dependence of elastic domain change on accumulated plastic strain. Because the elastic domain is constant during the plastic deformation process, except for initial loading, it is proposed to neglect the initial drop in the yield strength and consider a constant elastic domain throughout the whole deformation process. In this case, plastic deformation will be uniquely described by one component of the kinematic hardening variable (1).

\subsection{Stress-strain curve shape}

Accurate simulation of the stress-strain curve shape throughout the deformation process is one of the main aims of this study. It is established experimentally that the curve shape is affected by the phenomena of cyclic hardening and softening, asymmetry between tensile and compression loading and initial monotonic loading with the plateau behavior. It is also found that all stress-strain deformation curves are uniquely described by the two parameters logarithmic function with evolution rule (1). Therefore, the deformation processes are governed by two hardening parameters $A$ and $B$, which are functions of previously accumulation plastic strain $\bar{p}$ and plastic strain range $\bar{q}$. These variables are described by stepwise dependence with the use of the Dirac delta function. Therefore, they behave as constants during a tensile or compression step of a loading cycle. Subsequently, the parameters $A$ and $B$ also behave as constants during an active loading step and change their values only with reversal of loading. This is an important and convenient feature of the proposed model, as the calibration process for the shape of a single uniaxial stress-strain curve is uniquely described by only two parameters $A$ and $B$ without the effect of other parameters used for different cyclic plasticity phenomena. In general, particular values of $A$ and $B$ for a single uniaxial stress-strain curve can be determined using integrated form of the equation (1) for a tensile or compression part of loading:

$$
\begin{aligned}
& X^{t}=\frac{3}{2} B^{t} \ln \left[A^{t}\left(\varepsilon^{p}-\varepsilon_{0}^{p}\right)+\exp \left(-\frac{2\left(X_{0}-O\right)}{3 B^{t}}\right)\right]+O \\
& X^{c}=-\frac{3}{2} B^{c} \ln \left[-A^{c}\left(\varepsilon^{p}-\varepsilon_{0}^{p}\right)+\exp \left(\frac{2\left(X_{0}-O\right)}{3 B^{c}}\right)\right]+O
\end{aligned}
$$

where $X^{t}$ and $X^{c}$ are uniaxial back stresses for tension and compression defined as $X^{t}=\sigma-\sigma_{o}$ and $X^{c}=\sigma+\sigma_{o} ; \sigma$ is uniaxial stress measured experimentally; $\sigma_{o}$ is yield stress of the material; $X_{0}$ and $\varepsilon_{0}^{p}$ are uniaxial back stress and plastic strain at a moment of loading reversal.

For calibration purposes, it is convenient to place the back stress-strain curves with a reference point in the origin of the coordinate system as:

$$
\begin{gathered}
X^{t}=\frac{3}{2} B^{t} \ln \left(A^{t} \varepsilon^{p}+1\right) \\
X^{c}=-\frac{3}{2} B^{c} \ln \left(A^{c} \varepsilon^{p}+1\right)
\end{gathered}
$$


Table 1 - Summary of the constitutive model of cyclic plasticity

\begin{tabular}{l} 
Plastic flow \\
\hline Yield surface: \\
$f=\sqrt{\frac{3}{2}\left(S_{i j}-X_{i j}\right)\left(S_{i j}-X_{i j}\right)}-R-\sigma^{0}$ \\
Kinematic hardening: \\
$\dot{X}_{i j}=A B C D \exp \left(-\frac{2}{3} \frac{\left[X_{k l}-O_{k l}\right] n_{k l}}{B}\right) \dot{\varepsilon}_{i j}^{p}-\gamma\left(\left[X_{i j}-O_{i j}\right]-\frac{2}{3}\left[X_{k l}-O_{k l}\right] n_{k l} n_{i j}\right) \dot{p}$
\end{tabular}

Back stress reference tensor:

$O_{i j}=\frac{2 X^{y}-X^{h}}{2 X^{y}} \mu\left(X_{i j}+n_{i j} \beta-\bar{X}_{i j}\right)$

\section{Hardening surfaces}

Reference supersurface:

$F_{0}^{s}=\sqrt{\frac{3}{2} X_{i j} X_{i j}}-R_{s}-X_{y}$

Shifted supersurfaces:

$F_{i}^{s}=\sqrt{\frac{3}{2}\left(X_{i j}-\bar{c}_{i j}^{i}\right)\left(X_{i j}-\bar{c}_{i j}^{i}\right)}-R_{s}-X_{y}, i=1,2$

Radius of reference supersurface:

$\dot{R}_{s}=H\left(F_{0}^{s}\right) H\left(2 X_{h}-X_{y}\right) \frac{\dot{X}_{e q}}{\dot{p}} \dot{p}$

Centre of shifted supersurfaces:

$\dot{\bar{c}}_{i j}^{l}=\delta(G) \frac{d G}{d p}\left[\frac{R_{s}}{\underline{L}}\left(X_{i j}-\bar{X}_{i j}\right)+\bar{X}_{i j}-\bar{c}_{i j}^{l}\right] \mathbb{N}_{l} \dot{p}, l=1,2$

\section{Plastic strain range}

Global plastic strain range:

$\dot{\bar{q}}^{g l}=\delta\left(G^{p}\right) \frac{\dot{G}^{p}}{\dot{p}} H(-\mu)\left[\underline{\bar{q}}-\bar{q}^{g l}\right] \dot{p}$

Current plastic strain range:

$\dot{\bar{q}}=\delta\left(G^{p}\right) \frac{\dot{G}^{p}}{\dot{p}}\left[\frac{L^{p}}{2}-\bar{q}\right] \dot{p}+h_{1}\left[\bar{q}^{g l}-\bar{q}\right] \dot{p}$

Strain range dependence:

$d \mathrm{~A}^{s}=\alpha^{r}\left(\alpha^{s}-\mathrm{A}^{s}\right) d \bar{q}$

$d \mathrm{~B}^{s}=\beta^{r}\left(\beta^{s}-\mathrm{B}^{s}\right) d \bar{q}$

Global hardening parameters:

$\dot{A}^{g l}=\delta\left(G^{x}\right) \frac{\dot{G}^{x}}{\dot{p}} H(-\mu)\left[\underline{A}-\underline{A}^{g l}\right] \dot{p}$

$\dot{B}^{g l}=\delta\left(G^{x}\right) \frac{\dot{G}^{x}}{\dot{p}} H(-\mu)\left[\underline{B}-\underline{B}^{g l}\right] \dot{p}$

Current hardening parameters:

$\dot{A}=\mathrm{A}^{r}\left(\mathrm{~A}^{s}-A\right) \dot{\bar{p}}+\left[A^{g l}-\underline{A}-r^{A}(\bar{q})\right]\left(1-\left(\frac{L^{g l}}{R_{s}}\right)^{\kappa}\right) h_{1} \dot{p}+\delta\left(G^{x}\right) \frac{\dot{G}^{x}}{\dot{p}} H(-\mu) r^{A}(\bar{q}) \dot{p}$
$\dot{B}=\mathrm{B}^{r}\left(\mathrm{~B}^{s}-B\right) \dot{\bar{p}}+\left[B^{g l}-\underline{B}-r^{B}(\bar{q})\right]\left(1-\left(\frac{L^{g l}}{R_{s}}\right)^{\kappa}\right) h_{1} \dot{p}+\delta\left(G^{x}\right) \frac{\dot{G}^{x}}{\dot{p}} H(-\mu) r^{B}(\bar{q}) \dot{p}$ 
Table 1 (continued) - Summary of the constitutive model of cyclic plasticity

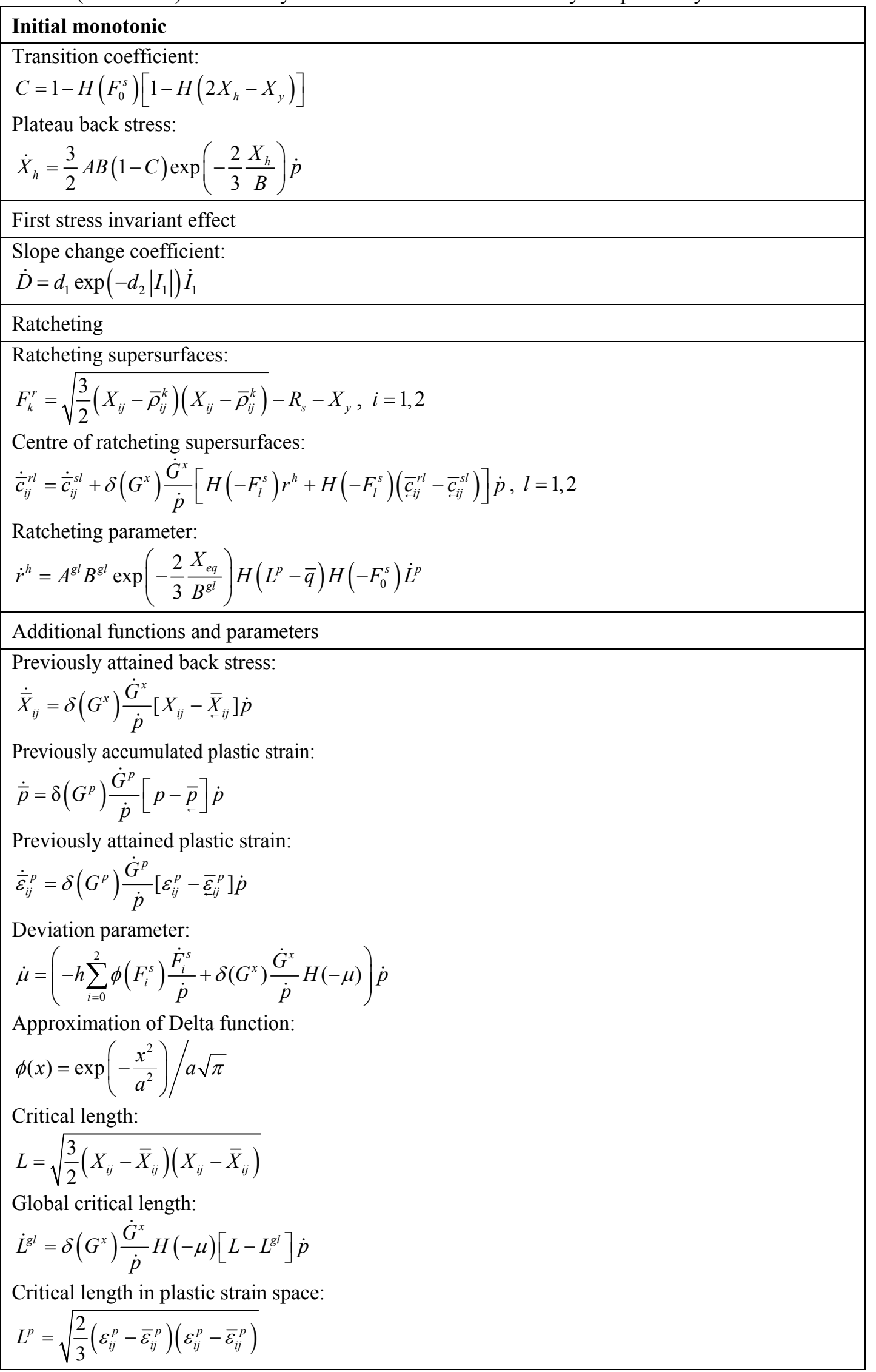


It is established experimentally that tensile and compression curves from a stress-strain curve of a stabilized hysteresis loop represented by $X^{t}$ and $X^{c}$ are not equal. Because this phenomenon is considered as the influence of the first stress invariant and modelled separately, it is proposed to consider the mean value of experimental $X^{t}$ and $X^{c}$ as a curve without the effect of the first stress invariant, as shown in Fig. 17. It is then defined for the case of stabilized hysteresis loop as:

$$
X=\frac{X^{t}-X^{c}}{2}
$$

The uniaxial back stress represented as (36) is believed to be exempt from the first invariant influence and can be experimentally evaluated from a torsion test with a pure shear type of loading. All stress-strain curves are defined by the uniaxial back stress as:

$$
X=\frac{3}{2} B \ln \left(A \varepsilon^{p}+1\right)
$$

\subsection{Calibration of hardening parameters}

To calibrate hardening parameters $A$ and $B$ to experimental stress-strain curves, different methods can be applied. Agius et al. (2017), Franulovic et al. (2014), Furukawa et al. (2002), Mahmoudi et al. (2011), Szeliga et al. (2004) used a calibration procedure which is based on optimization of an objective function, where material constants are parameters of the objective function. According to Franulovic et al. (2014), Szeliga et al. (2004), $A$ and $B$ parameters of the present model can be determined using the following objective function:

$$
F^{X}=\sqrt{\frac{1}{S} \sum_{i=1}^{s}\left(\frac{X_{i}^{\text {test }}-X_{i}^{\text {model }}(A, B)}{X_{i}^{\text {test }}}\right)^{2}}
$$

where $X_{i}^{\text {test }}$ is experimental values of back stress, $X_{i}^{\text {model }}(A, B)$ is calculated values of back stress according to (371) and $s$ is a number of experimental points.

Hardening parameters $A$ and $B$ of the kinematic hardening rule (1) are dependent on previously accumulated plastic strain and plastic strain range of cyclic loading by the evolution equations (23) and (24). Material constants $\alpha^{r}, \alpha^{s}, \beta^{r}, \beta^{s}, \mathrm{~A}^{r}$ and $\mathrm{B}^{r}$ together with its initial conditions $\left.\mathrm{A}^{s}\right|_{\bar{q}=0}=\mathrm{A}_{0}^{s},\left.\mathrm{~B}^{s}\right|_{\bar{q}=0}=\mathrm{B}_{0}^{s}$ , $\left.A\right|_{\bar{p}=0}=A_{0},\left.B\right|_{\bar{p}=0}=B_{0},\left.A^{g l}\right|_{\bar{p}=0}=A_{0}^{g l}$ and $\left.B^{g l}\right|_{\bar{p}=0}=B_{0}^{g l}$ should be determined.

Initial conditions for $\left.A\right|_{\bar{p}=0},\left.B\right|_{\bar{p}=0},\left.A^{g l}\right|_{\bar{p}=0}$ and $\left.B^{g l}\right|_{\bar{p}=0}$ are identified using the initial monotonic stressstrain curve. Figure 18 shows monotonic experimental curve after application of (36) to the monotonic curves obtained in tension and compression. Initial values of hardening parameters $A$ and $B$ are determined for the curve of initiation of plastic deformation (Curve 1 in Fig. 18). The reference point for this curve is taken to coincide with the plateau yield stress. Initial values for global hardening parameters are identified based on hardening which occurs after termination of the plateau region, as shown in Fig. 18 for Curve 2.

To identify material constants $\alpha^{r}, \alpha^{s}, \beta^{r}$ and $\beta^{s}$ with initial conditions $\mathrm{A}_{0}^{s}$ and $\mathrm{B}_{0}^{s}$ responsible for the saturation parameters $\mathrm{A}^{s}$ and $\mathrm{B}^{s}$ which depend on plastic strain range by (9) and (10), several stabilized hysteresis loops are required. For this material, 10 tests with 10 samples for different strain ranges, as shown in Fig. 1, have been shown to be sufficient to accurately determine the saturation behaviour of the hardening parameters. Under stabilized state of cyclic loading, equation (1) yields: 


$$
X=\frac{3}{2} \mathrm{~B}^{s} \ln \left(\mathrm{A}^{s} \varepsilon^{p}+1\right)
$$

where $\mathrm{A}^{s}$ and $\mathrm{B}^{s}$ are integrated from (9) and (10) as:

$$
\begin{aligned}
& \mathrm{A}^{s}=\alpha^{s}+\left(\mathrm{A}_{0}^{s}-\alpha^{s}\right) \exp \left(-\alpha^{r} \bar{q}\right) \\
& \mathrm{B}^{s}=\beta^{s}+\left(\mathrm{B}_{0}^{s}-\beta^{s}\right) \exp \left(-\beta^{r} \bar{q}\right)
\end{aligned}
$$

To be consistent with calibration of $\mathrm{A}^{s}$ and $\mathrm{B}^{s}$ for each plastic strain range, the following form of objective function is proposed:

$$
F^{s a t}=\sqrt{\sum_{j=1}^{l} w_{j}\left[\frac{1}{s} \sum_{i=1}^{s}\left(\frac{X_{i}^{\text {test }}\left(\bar{q}_{j}\right)-X_{i}^{\text {model }}\left(\alpha^{s}, \alpha^{r}, \beta^{s}, \beta^{r}, \mathrm{~A}_{0}^{s}, \mathrm{~B}_{0}^{s} ; \bar{q}_{j}\right)}{X_{i}^{\text {test }}\left(\bar{q}_{j}\right)}\right)^{2}\right]}
$$

where $\bar{q}_{j}$ is plastic strain amplitude of stabilized a hysteresis loop, $j$ is number of hysteresis loops (10 in the current case), $w_{j}$ is weight function to give different priority for different loops.

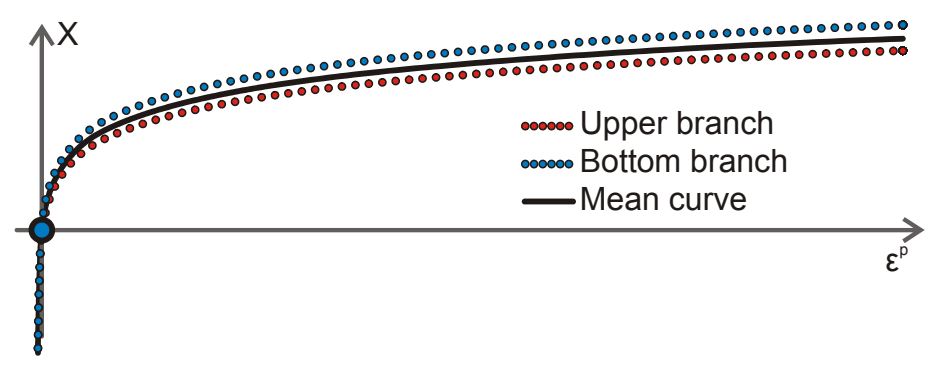

Fig. 17 - Mean curve definition
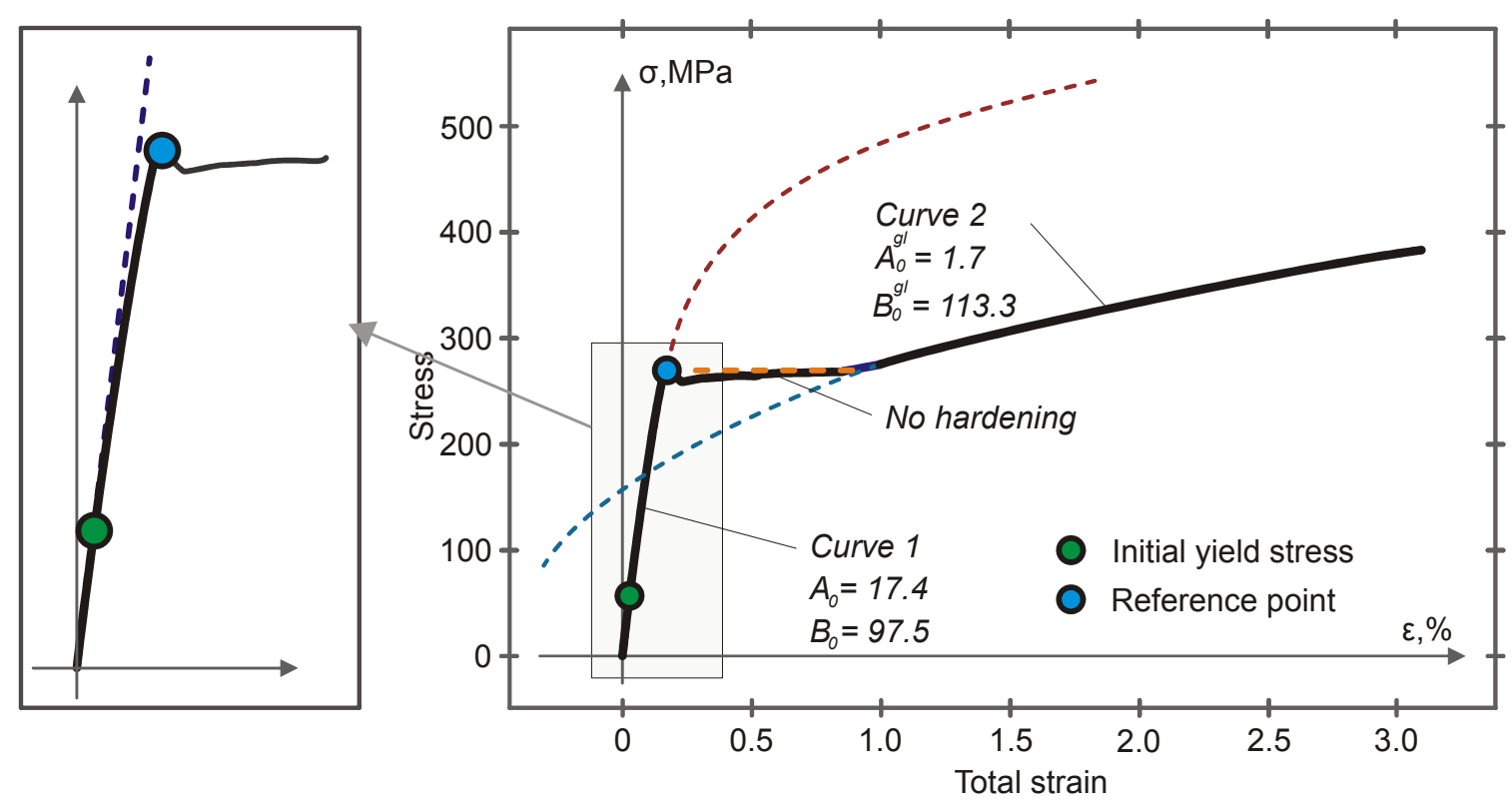

Fig. 18 - Determination of initial hardening parameters 
The use of the weight functions in the objective function (42) provides that stress-strain curves with larger plastic strain range have more importance for parameter identification as they have a longer strain path. To further identify parameters $\mathrm{A}^{r}$ and $\mathrm{B}^{r}$ which are responsible for the rate of the hardening saturation process, the integrated form of (7) and (8) which defines dependence on previously accumulated plastic strain is used in the following form:

$$
\begin{gathered}
A=\mathrm{A}^{s}+\left[A_{0}-\mathrm{A}^{s}\right] \frac{\exp \left(-\mathrm{A}^{r} \bar{p}\right)}{\exp \left(-\mathrm{A}^{r} \bar{p}_{0}\right)} \\
B=\mathrm{B}^{s}+\left[B_{0}-\mathrm{B}^{s}\right] \frac{\exp \left(-\mathrm{B}^{r} \bar{p}\right)}{\exp \left(-\mathrm{B}^{r} \bar{p}_{0}\right)}
\end{gathered}
$$

where $\bar{p}_{0}$ is initial value of previously accumulated plastic strain.

The same set of experiments used for determination of the constants for saturation of $A$ and $B$ can be used for the rate of saturation. A calibration process can be applied to find material constants for loading at each plastic strain range. Equations (43) and (44) are obtained by integrating equations (7) and (8) for the case of cyclic loading after the monotonic stress-strain curve. Transition from the monotonic curve is described by the transient functions (21) and (22). Therefore, the calibration process for hardening parameters should be applied to all curves from saturation at a plastic strain range except for the initial monotonic curve, the parameters of which having been previously defined. Equations (43) and (44) are substituted into (37) to obtain the back stress which defines the stress-strain curve shape of each curve from the saturation process. The objective function for determination of parameters $\mathrm{A}^{r}$ and $\mathrm{B}^{r}$ under loading at a given strain range is formulated as follows:

$$
F^{s a t}=\sqrt{\sum_{j=1}^{N} w_{j}\left[\frac{1}{s} \sum_{i=1}^{s}\left(\frac{X_{i}^{\text {test }}\left(\bar{p}_{j}\right)-X_{i}^{\text {model }}\left(\mathrm{A}^{r}, \mathrm{~B}^{r} ; \bar{p}_{j}\right)}{X_{i}^{\text {test }}\left(\bar{p}_{j}\right)}\right)^{2}\right]}
$$

where $\bar{p}_{j}$ is previously accumulated plastic strain defined at a moment of loading reversal, which can be calculated as $\bar{p}_{j}=\bar{p}_{j-1}+2 \bar{q}_{j-1} ; \mathrm{N}$ is a number of curves from the saturation process. It was established that full stabilization of hardening parameters is reached after $\sim 15$ cycles. Parameter $\kappa$, used to describe the change of global hardening parameters $A^{s m}$ and $B^{s m}$ after local cycling loading with small strain range, can be found from the tests with loading shown in Fig. 9.

\subsection{Initial monotonic behavior}

To define the parameters of the transient functions $r^{A}(\bar{q})$ and $r^{B}(\bar{q})$, which are introduced to describe an abrupt change of hardening for unloading from the initial monotonic stress-strain curve, unloading stages of the tests should be considered. The transient functions are the difference between predicted and actual hardening parameters:

$$
\begin{gathered}
r^{A}\left(\bar{q}_{i}\right)=A_{i}^{\text {mod }}-A_{i}^{a c t} \\
r^{B}\left(\bar{q}_{i}\right)=B_{i}^{\text {mod }}-B_{i}^{a c t}
\end{gathered}
$$


where $A_{i}^{\text {mod }}$ and $B_{i}^{\text {mod }}$ are predicted by model (7) and (8) and $A_{i}^{a c t}$ and $B_{i}^{a c t}$ are determined by calibration; $i$ is number of tests. The measured difference is then fitted by linear functions (21) and (22) with parameters $r_{1}^{A}, r_{2}^{A}, r_{1}^{B}$ and $r_{1}^{B}$.

\subsection{Asymmetry between tension and compression}

Accounting for the effect of the first stress invariant is important for all curves from the deformation process. Therefore, before determination of the hardening parameters for stress-strain curves, it is required to establish numerical values for material parameters from the evolution law for the first stress invariant influence. To identify material parameters, one hysteresis loop with a largest considered strain range is used. The following system of differential equations governs the material behaviour for the case when tensile and compression curves are plotted with a reference point at the origin of the coordinate system:

$$
\begin{gathered}
\dot{X}_{i j}=A B D \exp \left(-\frac{2}{3} \frac{\left(X_{k l}-O_{k l}\right) n_{k l}}{B}\right) \dot{\varepsilon}_{i j}^{p} \\
\dot{D}=d_{1} J \exp \left(-d_{2}\left|I_{1}\right|\right) \dot{p}
\end{gathered}
$$

In the uniaxial case of tension and compression, the system (48) - (49) transforms to the following:

$$
\begin{gathered}
\dot{X}=\frac{3}{2} A B D \exp \left(s \frac{2 X}{3 B}\right) \dot{\varepsilon}^{p} \quad\left\{\begin{array}{c}
s=-1 \text { for tension } \\
s=1 \text { for compression }
\end{array}\right. \\
\dot{D}=d_{1} \exp \left(-d_{2}\left|X+\sigma_{0}\right|\right) \frac{\dot{X}}{\dot{p}} \dot{p}
\end{gathered}
$$

The second equation of the system can be solved separately giving the following expression with initial conditions of $D(0)=1$ :

$$
D=\frac{s d_{1}}{d_{2}}\left[1-\exp \left(-s d_{2}\left[X+\sigma_{0}\right]\right)\right]
$$

Substitution of (52) into the first equation of the system gives:

$$
\dot{X}=\frac{3}{2} A B \frac{s d_{1}}{d_{2}}\left[1-\exp \left(-s d_{2}\left[X+\sigma_{0}\right]\right)\right] \exp \left(s \frac{2 X}{3 B}\right) \dot{\varepsilon}^{p}
$$

Equation (53) has an analytical solution which is not given here due to size constraint. Alternatively, this equation can be solved numerically or taken in an approximated form as:

$$
X=\frac{3}{2} B \frac{s d_{1}}{d_{2}}\left[1-\exp \left(-s d_{2}\left[\ln \left(A \varepsilon^{p}+1\right)+\sigma_{0}\right]\right)\right] \ln \left(A \varepsilon^{p}+1\right)
$$

Parameters $A$ and $B$ are determined from the mean curve (36). The unknown parameters $d_{1}$ and $d_{2}$ are identified with the use of the following objective function:

$$
F^{X}=\sqrt{\frac{1}{s} \sum_{i=1}^{s}\left(\frac{X_{i}^{\text {test }}-X_{i}^{\text {model }}\left(d_{1}, d_{2}\right)}{X_{i}^{\text {test }}}\right)^{2}}
$$

The whole set of identified material parameters is presented in Table 2. 
Table 2 - Identified material constants of the proposed plasticity model for S355J2 steel

\begin{tabular}{|c|c|}
\hline Basic parameters: & $\sigma^{0}=55 M P a, X^{y}=200 M P a$ \\
\hline Hardening parameters: & $\begin{array}{l}\alpha^{s}=78.5, \alpha^{r}=4.6, \beta^{s}=61.9 \mathrm{MPa}, \beta^{r}=2.0, \mathrm{~A}^{r}=0.24, \mathrm{~B}^{r}=0.4, \kappa=0.05, \\
A_{0}^{g l}=1.7, B_{0}^{g l}=113.3 \mathrm{MPa}, A_{0}=17.4, B_{0}=97.5 \mathrm{MPa}\end{array}$ \\
\hline Initial monotonic behaviour: & $r_{1}^{A}=-10.1, r_{2}^{A}=11.9, r_{1}^{B}=16.2, r_{2}^{B}=19.0$ \\
\hline First stress invariant effect: & $d_{1}=0.002, d_{2}=0.01$ \\
\hline Ratcheting: & $a=15$ \\
\hline Nonproportional loading & $\gamma=5000$ \\
\hline
\end{tabular}

\section{Numerical verification}

In this section, examples of application of the developed constitutive model to the uniaxial loading of the investigated S355J2 steel and to nonproportional tension-torsion loading of a copper are presented and discussed. The specific form of the developed set of differential equations shown in Table 1 allows evaluation of analytical solutions for a uniaxial cyclic loading problem when it is divided on steps of tensile and compression loading. In this case, the Dirac delta function is integrated to the Heaviside step function, where quantities from the previous step are used as initial conditions for subsequent steps. Appropriate choice of approximation of the Dirac delta function for ratcheting and mean stress relaxation also allows analytical solutions for the uniaxial cyclic plasticity problems. Alternatively, all the differential equations from Table 1 can be efficiently solved numerically with no need for splitting the problem into steps, where the Dirac delta function is approximated by (28) with small finite values of $a$ parameter. Some equations from Table 1 are delay deferential equations, where the time delay can be chosen as any duration smaller than a loading cycle but larger than a time increment used in a computational algorithm. Most commercial mathematical software is able to solve first order delay differential equations, for example by the Fehlberg fourth-fifth order RungeKutta method. Therefore, no special numerical algorithm for solving the set of the developed equations from Table 1 is required; all equations can be inserted into a commercial mathematical package and solved automatically by existing numerical algorithms. This approach can be used to solve one-dimensional uniaxial and two- and three-dimensional multiaxial problems, where differential equations of equilibrium are not involved, such as in case of tension-compression with torsion of a thin-walled tube under internal pressure. More general 3D problems are solved by the Finite Element Method, where integration of differential equations from Table 1 can be based on backward Euler discretization with a radial return mapping algorithm such as used by Kobayashi and Ohno (2002). In this case, a problem is divided on time or load steps and all quantities defined with time delay are computed from the previous integration step if the conditions for loading reversal or change of strain range are satisfied.

Figure 19 shows the stabilized cyclic stress-strain hysteresis loops under loading with different strain ranges. Numerical simulation of this responses is done with the use of the full developed model as well as a simplified model, where kinematic hardening is considered without hardening supersurfaces, plastic strain range dependence and first stress invariant effect in the form of:

$$
\dot{X}_{i j}=A B \exp \left(-\frac{2}{3} \frac{X_{k l} n_{k l}}{B}\right) \dot{\varepsilon}_{i j}^{p}
$$

It is shown that the simplified model, which is calibrated to the bottom stress-strain curve from the hysteresis loop with the largest strain range, gives reasonable prediction of all stress-strain curves with certain deviations from experimental results for smaller strain ranges and curves from upper branches of the hysteresis loops. Accounting for the phenomena of plastic strain range dependence and first stress invariant in the full model makes predictions conform well to the experiments. 


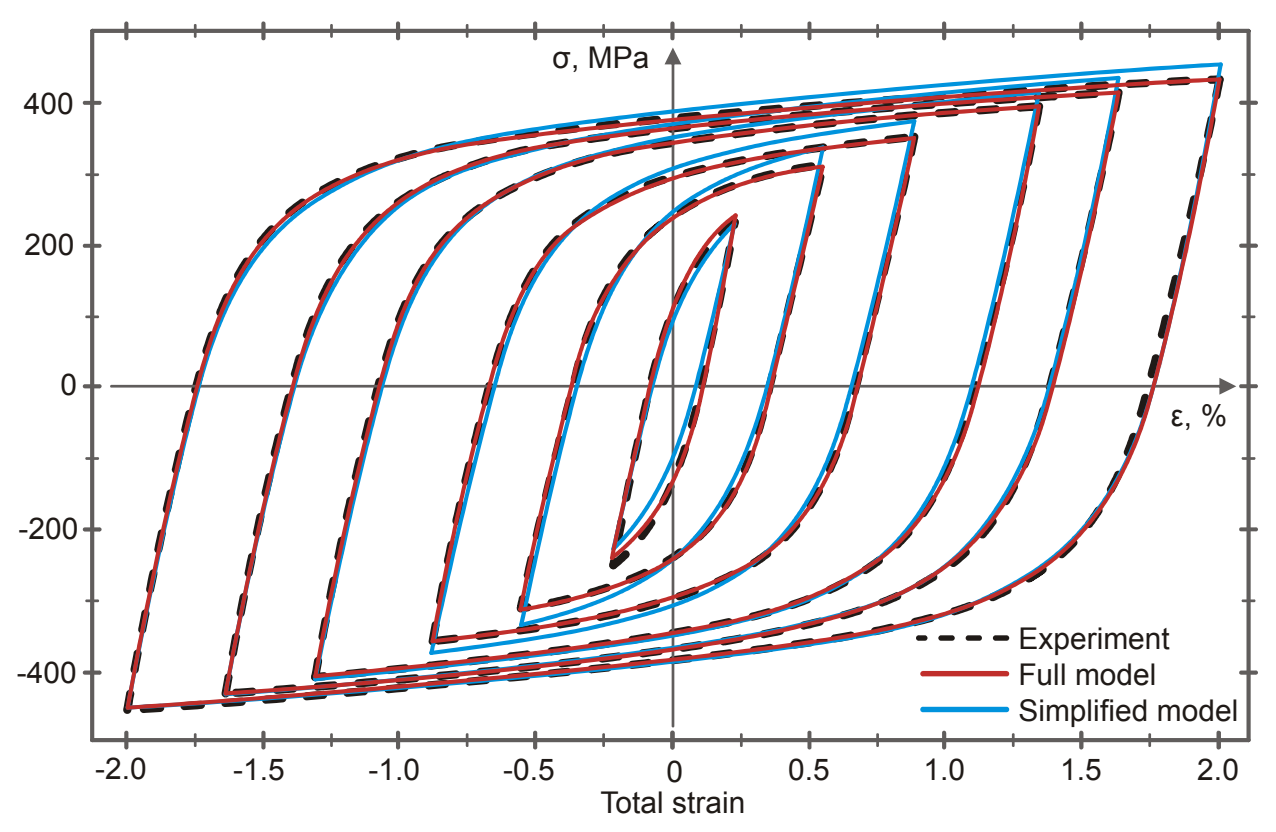

Fig. 19 - Stabilized hysteresis loops of S355J2 steel with modelling

Figure 20 shows application of the full and simplified models to the stabilization process of deformation under fixed strain range of $1.3 \%$ starting from the monotonic behaviour. It is shown that the simplified model calibrated to either initial monotonic or stabilized stress-strain curves is not able to properly describe transition between curves during the saturation process. As shown and discussed by Hassan et al. (2008) and Krishna et al. (2009), simulation of the stress-strain curve shapes of the initial monotonic and subsequent cyclic curves by one set of calibrated parameters is a challenging task. Therefore, two different sets of material parameters calibrated to both monotonic and cyclic responses are usually used in practice. To resolve this problem and use only one calibrated set of parameters, Krishna et al. (2009) proposed a special form of the isotropic hardening variable, utilizing an additional term for reduction of the yield surface size during initial monotonic loading. This approach however may not be convenient for certain materials, S355J2 for example, where it is established in Part I that yield surface elastic domain does not change during plastic deformation, including monotonic loading, except for a small initial strain region. The proposed model with full formulation precisely simulates the stress-strain curve shapes of all curves of the deformation process, including initial monotonic behaviour with the plateau, with only one set of calibrated parameters and kinematic hardening only.

High accuracy of matching the experimental results for initial monotonic behaviour with the plateau by one constitutive model is achieved by a special form of strain range dependence incorporated into the hardening parameters together with introduction of the transient functions for modelling of transition from the initial monotonic stress-strain curve. Figure 21 shows the response of S355J2 steel to cyclic loading with increasing loading amplitude, demonstrating the hardening process from the initial virgin state of the material. The difficulty in modelling this behaviour is the large difference between the hardening parameters of the initial monotonic curve and subsequent curves of cyclic loading. As seen from Fig. 21, the proposed model is able to accurately represent loading-reloading cycles from initial monotonic stress-strain curve with the plateau of S355J2 steel.

Simulation of the behaviour of transition from the initial monotonic stress-strain curve is also presented the for S460N steel. Performance of the proposed model is compared with Döring et al. (2003) model, which is based on A-F type of kinematic hardening with Chaboche back stress decomposition. It is shown that the proposed model gives better representation of stress-strain curves except for a small region at the initiation of the plateau. The proposed model also has better description of transition between the elastic and elastic-plastic 
states of unloading curves. This is achieved by choosing a low value of the elastic limit allowing nonlinear unloading behaviour to start earlier than in the case of Döring et al. (2003) model.

The material constants $\alpha^{r}, \alpha^{s}, \beta^{r}, \beta^{s}, \mathrm{~A}^{r}$ and $\mathrm{B}^{r}$ of the hardening parameters of the proposed model are initially calibrated to stress-strain curves from loading where the saturation process occurs at fixed strain ranges. It is necessary to verify performance of the model for the case when loading reversals occur randomly under different strain ranges without stabilization at a certain strain range. An example of such a loading program is shown in Fig. 23, where the strain range is increased step by step. It is shown that the stepwise representation of the hardening process achieved by the Dirac delta function approach is valid for this type of loading program. It is demonstrated that all stress-strain curves from cyclic loading can be uniquely described by two parameters $A$ and $B$, where their values are determined by plastic strain range and amount of previously accumulated plastic strain of a current loading state.

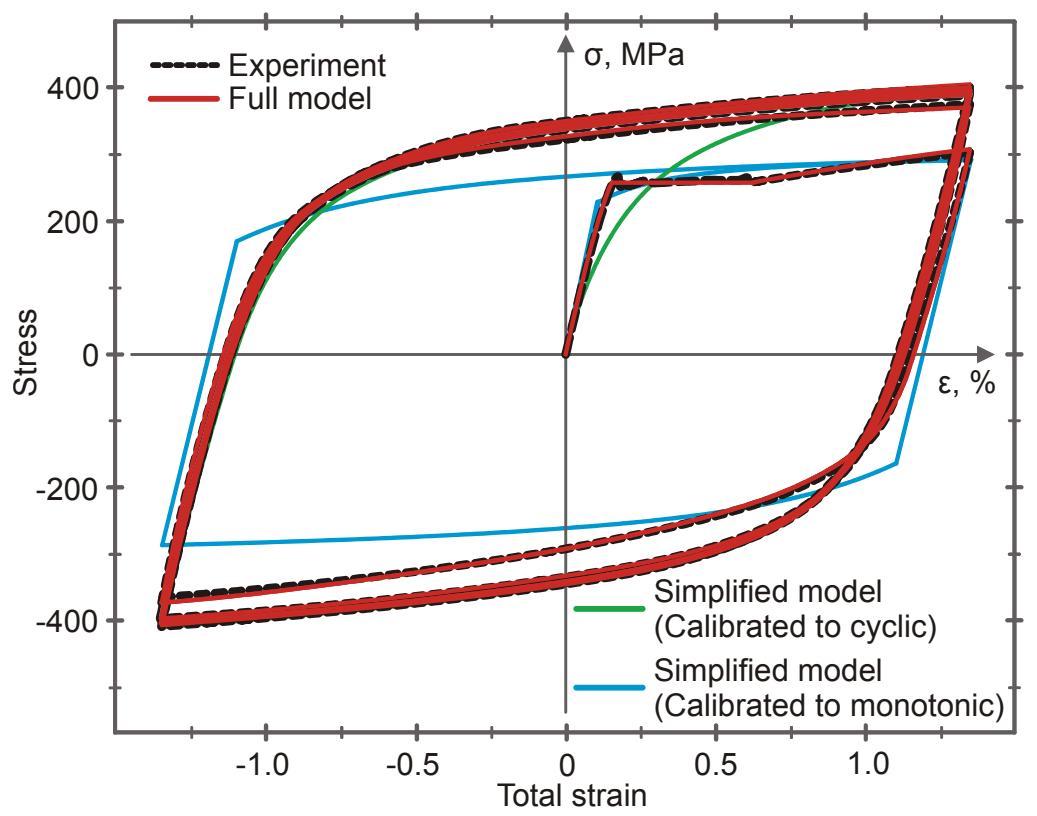

Fig. 20 - Stress-strain curve of loading at $1.3 \%$ of strain range with modelling for S355J2 steel

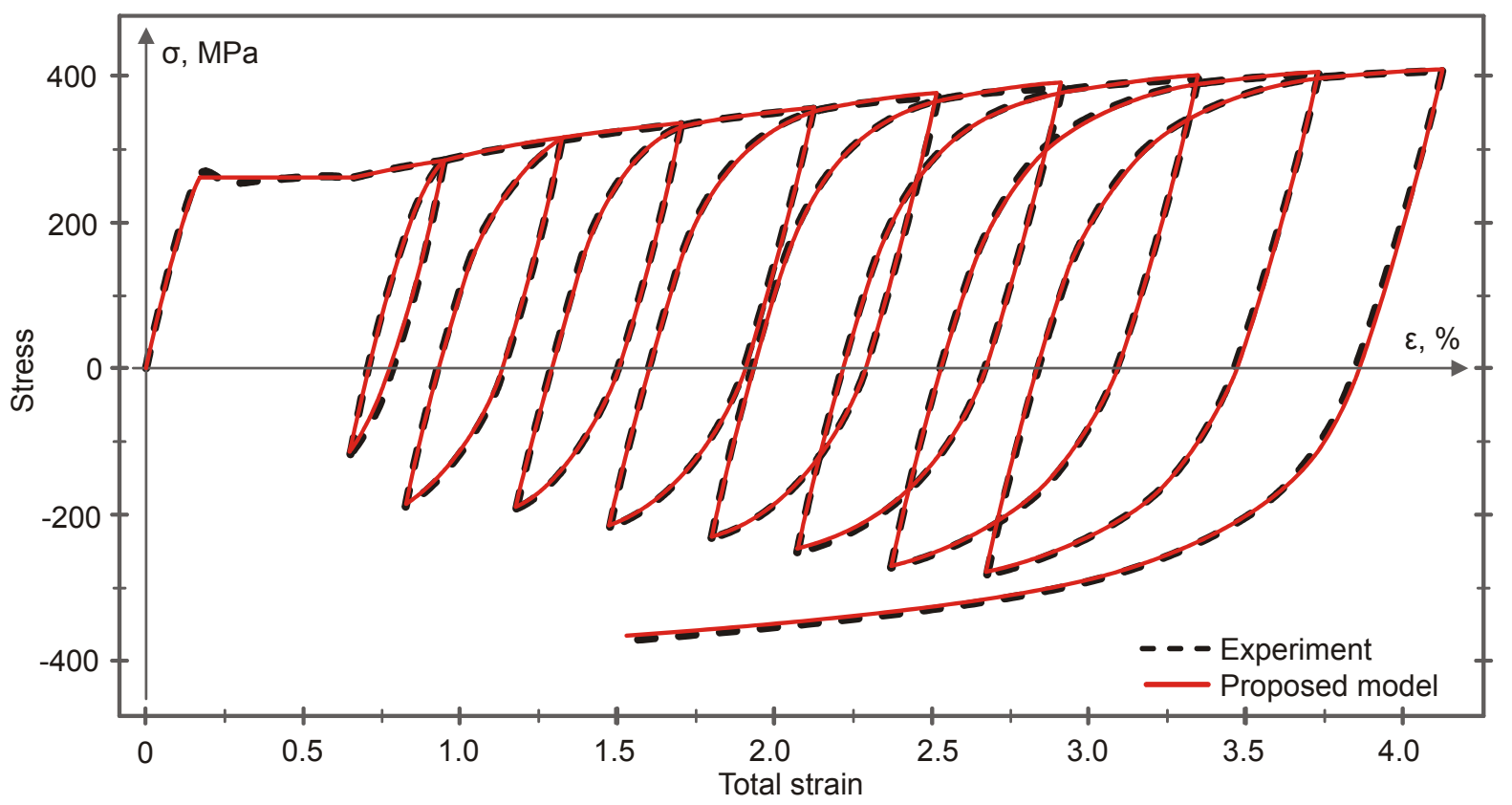

Fig. 21 - Stress-strain curve of S355J2 steel with increasing loading 


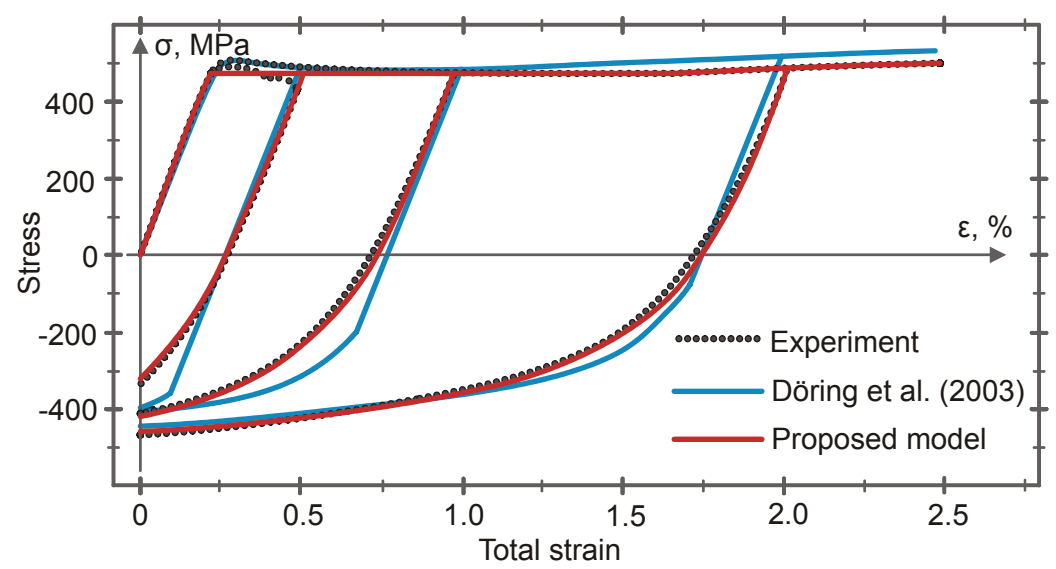

Fig. 22 - Loading-unloading stress-strain curves of S460N steel (experimental results from (Döring et al., 2003))

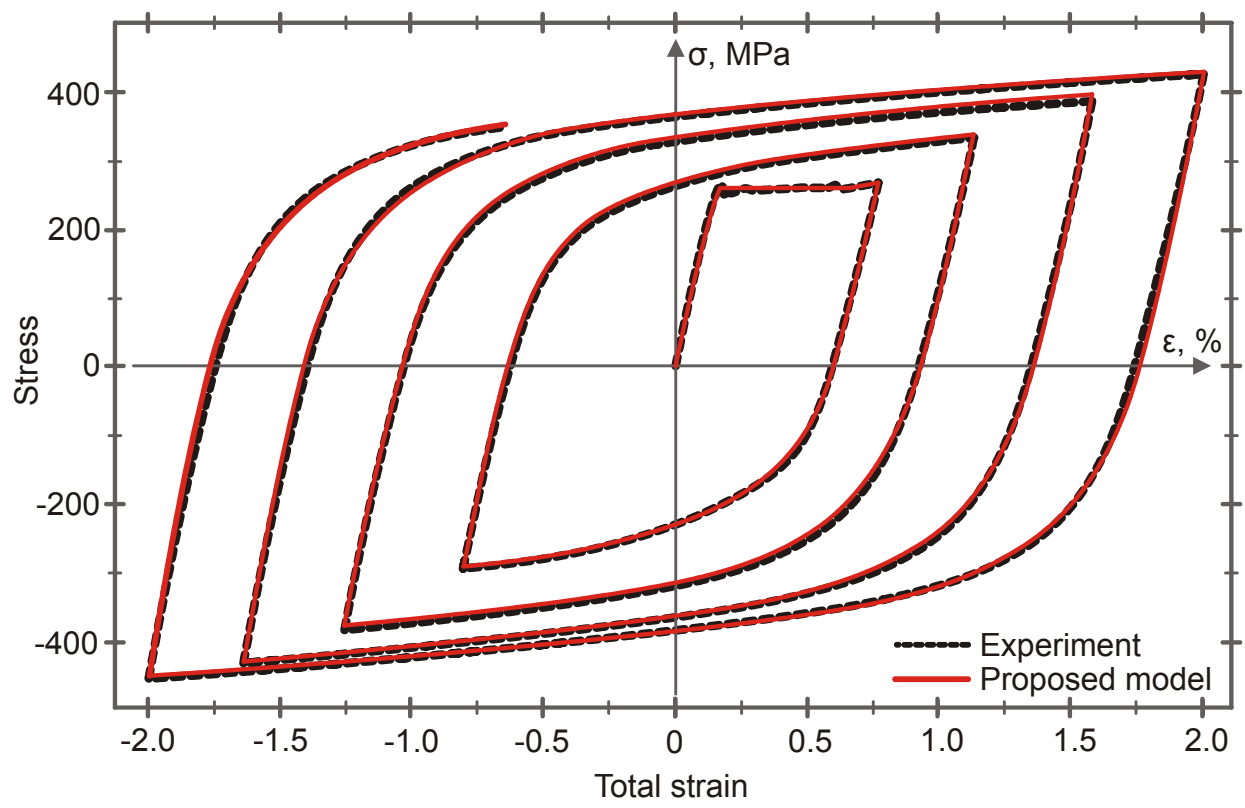

Fig. 23 - Stress-strain curve of S355J2 steel under randomly increasing strain range loading with modelling

It has been shown that the proposed model in the form of (1) provides very good capability in accurate modelling of experimental stress-strain curves for S355J2 steel, as well as other materials, for the case of proportional loading condition. To include the case of non-proportional loading with a directional behaviour of back stress, (1) is modified to the following form:

$$
\dot{X}_{i j}=A B C D \exp \left(-\frac{2}{3} \frac{\left[X_{k l}-O_{k l}\right] n_{k l}}{B}\right) \dot{\varepsilon}_{i j}^{p}-\gamma\left(\left[X_{i j}-O_{i j}\right]-\frac{2}{3}\left[X_{k l}-O_{k l}\right] n_{k l} n_{i j}\right) \dot{p}
$$

Experimental verification of the proposed model for the case of nonproportional loading is considered for annealed copper, experimentally investigated in (Lamba and Sidebottom, 1978a, b). The proposed model for cyclic hardening/softening considers change of hardening parameters, which only occurs during proportional loading such as variation between tensile and compression loading. To incorporate nonproportional cyclic hardening, a nonproportional parameter proposed by Tanaka (1994) should be incorporated into hardening parameters $A$ and $B$. This is not yet implemented in the proposed model. However, a nonproportional response can still be verified using a cyclically stable experiment performed for copper according to the loading program shown in Fig. 24a (Lamba and Sidebottom, 1978b). For modelling, three cases are considered: the Chaboche model with 2 back stresses and the proposed model with $\gamma=0$ and $\gamma=5000$. Figure $24 \mathrm{~b}$ shows that the first proportional step of the loading program is simulated well by the three models, 
as no non-proportionality is involved. Figure $24 \mathrm{c}$ suggests that both the Chaboche model and the proposed model with $\gamma=5000$ simulate the nonproportional experimental response of copper reasonably accurately. It is noted that for a cyclically stable material, the basic formulation of the Chaboche model gives accurate prediction of both the proportional and nonproportional responses. The proposed model has shown advantages in accurate simulation of cyclically non-stable materials for proportional loading. Therefore, the model is expected to give accurate simulation response under nonproportional loading conditions after adding the nonproportional cyclic hardening parameter. This has yet to be extensively verified experimentally for different material with different nonproportional loading conditions. The proposed model with $\gamma=0$, which uses only the first term of the right hand side of equation (57), is incorrect in simulating nonproportional behaviour. In this case, back stress can only evolve in the direction of plastic strain tensor. Therefore, the presence of the second term in equation (57) is necessary for accurate simulation of nonproportional hardening. The considered example of the steady stable cyclic behaviour of copper shows correct nonproportional simulation of the proposed model in the form (57). One benefit of such representation of the kinematic hardening rule is that if only proportional cyclic loading is considered, the second term in equation (57) can be ignored and the constitutive model is simplified.
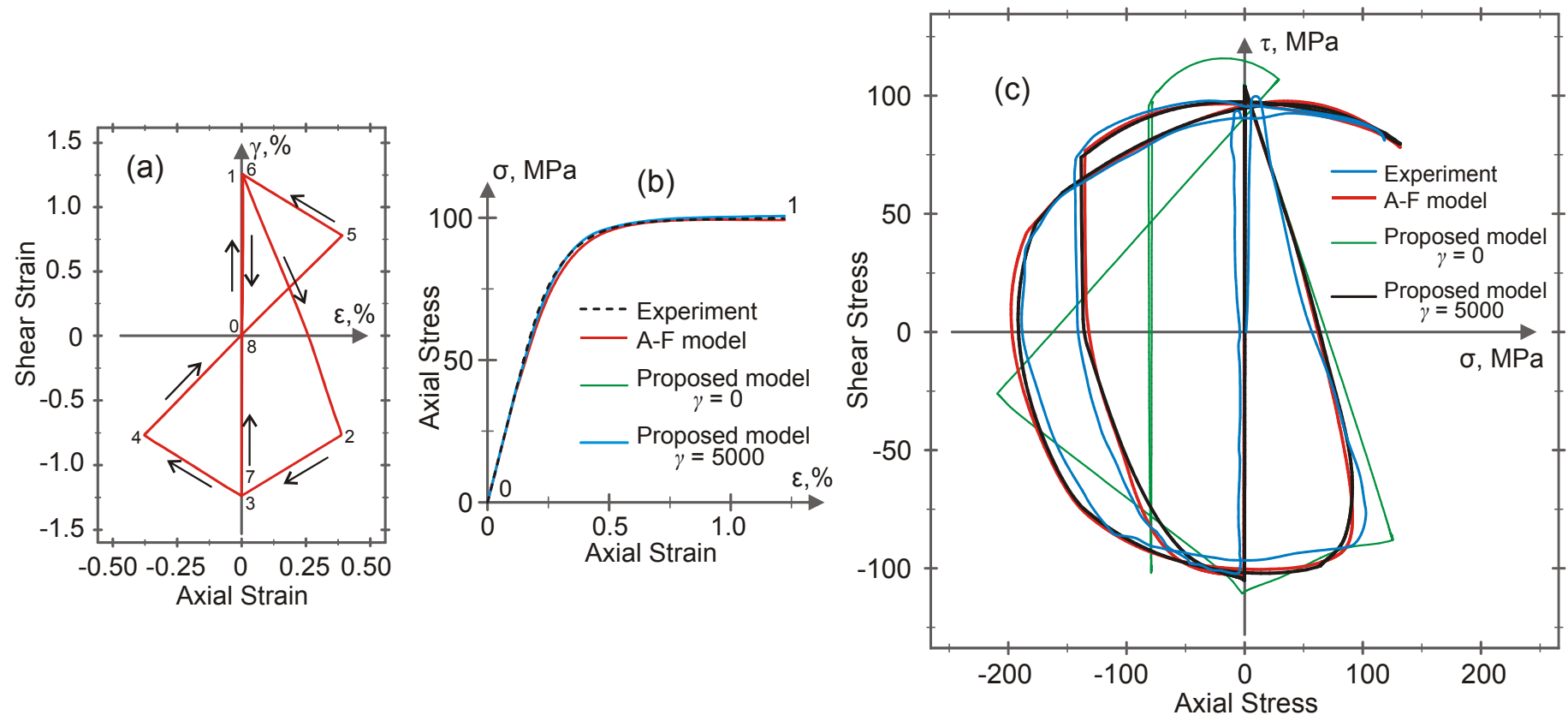

Fig. 24 - Experiment and simulation of tension-torsion nonproportional cyclic loading for copper (experimental results from (Lamba and Sidebottom, 1978b)); (a) strain controlling loading program, (b) axial stress vs. axial strain response during the first step of loading and (c) axial stress vs. shear stress response during nonproportional loading

\section{Conclusions}

Part II of this study completes the development of the model introduced in Part I by consideration of the cyclic hardening and softening behaviours and ratcheting of materials. The proposed approach of Dirac delta functions is extended to description of plastic strain range change and variation of hardening parameters with accumulation of plastic strain under different strain amplitudes. The Dirac delta function framework introduces certainty in the definition of plastic strain range variation which is especially useful for cases of non-proportional loading conditions. For the first time, plastic strain range dependence of hardening parameters is presented as a function of both plastic strain range and accumulated plastic strain allowing realistic simulation of material behavior of cyclic hardening and softening irrespectively of loading programs and number of loading reversals under different strain ranges. In the case of the investigated S355J2 steel, the hardening parameters of the proposed kinematic hardening model showed saturation of their values with increasing plastic strain range as well as saturation under fixed plastic strain range with accumulating of 
plastic strain. Therefore, two saturation type functions with 6 material constants are used to describe cyclic hardening/softening of this material.

It is found that cyclic hardening behavior occurs with increasing plastic strain range and cyclic softening happens with its decreasing. The proposed model fully describes the whole range of loading conditions including the initial monotonic behavior by the introduced dependence of kinematic hardening parameters on plastic strain range and accumulated plastic strain. Dividing the deformation process on states of global and local hardening allows to more accurately describe the cyclic hardening and softening behaviour which occurs during transitions between loading at small and large strain ranges. Regardless of a number of increasing or decreasing strain range of loading, saturation values of hardening parameters of the proposed model are precisely defined by the master curve of Fig. 3, whereas their saturation process is described by dependence of previously accumulated plastic strain.

Ratcheting behaviour of materials is modelled mathematically with the use of the Dirac delta function approach, where its approximated form is efficiently used to simulate the deviation effect of stress path tending to close the hysteresis loop. The predictive ability of the developed cyclic plastic model for ratcheting simulation is analysed with emphasis on the importance of consideration of accurate curve shape modelling during cyclic the hardening/softening process. The effect of decreasing ratcheting rate with accumulation of plastic strain is modelled by introduction of two additional ratcheting supersurfaces which are associated with shifted supersurfaces. Evolution of these supersurfaces provides their relaxation on the reference supersurface, which simulates decrease in ratcheting rate, cyclic mean stress relaxation and some shakedown effects. It is observed experimentally that the ratcheting phenomenon is affected by cyclic hardening and softening, such that hardening increases ratcheting rate and softening decreases it. Therefore, realistic modelling of the cyclic hardening/softening behavior is crucial in accurate prediction of the ratcheting rate. The proposed model shows promising results of the simulation of S355J2 steel under different loading conditions for both stabilized and non-stabilized states. The approach of ratcheting supersurfaces provides some flexibility with the choice of their evolution rules which can be used for modelling under a wider range of loading programs including non-proportional loading conditions.

In addition to the developed theoretical framework, a detailed calibration procedure to determine material constants of the model for S355J2 steel was presented. The specific form of the developed model allows consideration of most of cyclic plasticity phenomena independently of each other. Subsequently, sets of material constants are effectively determined per considered phenomenon in a clear and concise manner. Determination of material parameters for cyclic hardening/softening behavior with strain range dependence is based not on variation of peak stresses, but rather on actual shape of every stress-strain curve of the deformation process. The model is fully defined with the use of 17 material constants and 4 initial conditions in total, which is commensurate with accuracy of the model in matching experimental results of the phenomena of cyclic hardening/softening, ratcheting, mean stress relaxation and difference between tensile and compression behaviour within the whole range of loading including the initial monotonic behavior with the yield plateau. This is demonstrated in the numerical verification of the model for the cases of standard loading programs as well as programs with specific loading conditions including random loading. With the use of the unified theoretical set of developed equations, the model is capable of predicting accurate material responses under different cyclic loading programs, which is very useful in practical applications to problems with both steady loading conditions where an accurate stabilized behaviour is required and non-steady conditions with transition from the initial monotonic to cyclic behaviours.

From a practical perspective, the fact that most of internal variables of the Dirac delta function approach behave with stepwise dependence during a proportional loading step makes the model easy in implementation as all evolutional equations can be integrated analytically. Therefore, numerical schemes used for solving 
engineering problems by the proposed model are similar to those developed for A-F type kinematic hardening models.

\section{Acknowledgments}

This project has received funding from the European Union's Horizon 2020 research and innovation programme under the Marie Sklodowska-Currie grant agreement No 643159.

\section{References}

Abdel-Karim, M., 2009. Modified kinematic hardening rules for simulations of ratchetting. International Journal of Plasticity 25, 1560-1587.

Abdel-Karim, M., 2010. An evaluation for several kinematic hardening rules on prediction of multiaxial stresscontrolled ratchetting. International Journal of Plasticity 26, 711-730.

Abdel-Karim, M., Ohno, N., 2000. Kinematic hardening model suitable for ratchetting with steady-state. International Journal of Plasticity 16, 225-240.

Agius, D., Kajtaz, M., Kourousis, K.I., Wallbrink, C., Wang, C.H., Hu, W., Silva, J., 2017. Sensitivity and optimisation of the Chaboche plasticity model parameters in strain-life fatigue predictions. Materials \& Design 118, 107-121.

Bari, S., Hassan, T., 2000. Anatomy of coupled constitutive models for ratcheting simulation. International Journal of Plasticity 16, 381-409.

Bari, S., Hassan, T., 2001. Kinematic hardening rules in uncoupled modeling for multiaxial ratcheting simulation. International Journal of Plasticity 17, 885-905.

Chaboche, J.L., 1979. Modelization on the Strain Memory Effect on the Cyclic Hardening of 316 Stainless Steel. Trans. of the 5th Int. Conf. on SMiRT 11.

Chaboche, J.L., 1991. On some modifications of kinematic hardening to improve the description of ratchetting effects. International Journal of Plasticity 7, 661-678.

Chaboche, J.L., 2008. A review of some plasticity and viscoplasticity constitutive theories. International Journal of Plasticity 24, 1642-1693.

Chaboche, J.L., Gaubert, A., Kanouté, P., Longuet, A., Azzouz, F., Mazière, M., 2013. Viscoplastic constitutive equations of combustion chamber materials including cyclic hardening and dynamic strain aging. International Journal of Plasticity 46, 1-22.

Chen, X., Jiao, R., 2004. Modified kinematic hardening rule for multiaxial ratcheting prediction. International Journal of Plasticity 20, 871-898.

Dafalias, Y.F., Popov, E.P., 1975. A model of nonlinearly hardening materials for complex loading. Acta Mechanica 21, 173-192.

Döring, R., Hoffmeyer, J., Seeger, T., Vormwald, M., 2003. A plasticity model for calculating stress-strain sequences under multiaxial nonproportional cyclic loading. Computational Materials Science 28, 587-596.

Franulovic, M., Basan, R., Krizan, B., 2014. Kinematic Hardening Parameters Identification with Respect to Objective Function. International Journal of Mechanical, Aerospace, Industrial, Mechatronic and Manufacturing Engineering 88, $686-690$.

Furukawa, T., Sugata, T., Yoshimura, S., Hoffman, M., 2002. An automated system for simulation and parameter identification of inelastic constitutive models. Computer Methods in Applied Mechanics and Engineering 191, 22352260.

Guionnet, C., 1992. Modeling of Ratchetting in Biaxial Experiments. Journal of Engineering Materials and Technology 114, 56-62.

Hassan, T., Corona, E., Kyriakides, S., 1992. Ratcheting in cyclic plasticity, part II: Multiaxial behavior. International Journal of Plasticity 8, 117-146.

Hassan, T., Kyriakides, S., 1992. Ratcheting in cyclic plasticity, part I: Uniaxial behavior. International Journal of Plasticity 8, 91-116.

Hassan, T., Kyriakides, S., 1994a. Ratcheting of cyclically hardening and softening materials: I. Uniaxial behavior. International Journal of Plasticity 10, 149-184.

Hassan, T., Kyriakides, S., 1994b. Ratcheting of cyclically hardening and softening materials: II. Multiaxial behavior. International Journal of Plasticity 10, 185-212. 
Hassan, T., Taleb, L., Krishna, S., 2008. Influence of non-proportional loading on ratcheting responses and simulations by two recent cyclic plasticity models. International Journal of Plasticity 24, 1863-1889.

Kang, G., Ohno, N., Nebu, A., 2003. Constitutive modeling of strain range dependent cyclic hardening. International Journal of Plasticity 19, 1801-1819.

Khutia, N., Dey, P.P., Hassan, T., 2015. An improved nonproportional cyclic plasticity model for multiaxial low-cycle fatigue and ratcheting responses of 304 stainless steel. Mechanics of Materials 91, 12-25.

Kobayashi, M., Ohno, N., 2002. Implementation of cyclic plasticity models based on a general form of kinematic hardening. International Journal for Numerical Methods in Engineering 53, 2217-2238.

Krishna, S., Hassan, T., Ben Naceur, I., Saï, K., Cailletaud, G., 2009. Macro versus micro-scale constitutive models in simulating proportional and nonproportional cyclic and ratcheting responses of stainless steel 304. International Journal of Plasticity 25, 1910-1949.

Lamba, H.S., Sidebottom, O.M., 1978a. Cyclic Plasticity for Nonproportional Paths: Part 1-Cyclic Hardening, Erasure of Memory, and Subsequent Strain Hardening Experiments. Journal of Engineering Materials and Technology 100, 96103.

Lamba, H.S., Sidebottom, O.M., 1978b. Cyclic Plasticity for Nonproportional Paths: Part 2-Comparison With Predictions of Three Incremental Plasticity Models. Journal of Engineering Materials and Technology 100, 104-111. Landgraf, R.W., Morrow, J.-D., Endo, T., 1969. Determination of the cyclic stress-strain curve. J. Mater. - JMLSA 4, 176 $-188$.

Mahmoudi, A.H., Pezeshki-Najafabadi, S.M., Badnava, H., 2011. Parameter determination of Chaboche kinematic hardening model using a multi objective Genetic Algorithm. Computational Materials Science 50, 1114-1122.

McDowell, D.L., 1985a. A Two Surface Model for Transient Nonproportional Cyclic Plasticity, Part 1: Development of Appropriate Equations. Journal of Applied Mechanics 52, 298-302.

McDowell, D.L., 1985b. A Two Surface Model for Transient Nonproportional Cyclic Plasticity, Part 2: Comparison of Theory With Experiments. Journal of Applied Mechanics 52, 303-308.

McDowell, D.L., 1987. An Evaluation of Recent Developments in Hardening and Flow Rules for Rate-Independent, Nonproportional Cyclic Plasticity. Journal of Applied Mechanics 54, 323-334.

Nouailhas, D., Cailletaud, G., Policella, H., Marquis, D., Dufailly, J., Lieurade, H.P., Ribes, A., Bollinger, E., 1985. On the description of cyclic hardening and initial cold working. Engineering Fracture Mechanics 21, 887-895.

Ohno, N., 1982. A Constitutive Model of Cyclic Plasticity With a Nonhardening Strain Region. Journal of Applied Mechanics 49, 721-727.

Ohno, N., Wang, J.D., 1993a. Kinematic hardening rules with critical state of dynamic recovery, part I: formulation and basic features for ratchetting behavior. International Journal of Plasticity 9, 375-390.

Ohno, N., Wang, J.D., 1993b. Kinematic hardening rules with critical state of dynamic recovery, part II: Application to experiments of ratchetting behavior. International Journal of Plasticity 9, 391-403.

Ohno, N., Yamamoto, R., Sasaki, T., Okumura, D., 2018. Resetting scheme for plastic strain surface in constitutive modeling of cyclic plasticity. ZAMM - Journal of Applied Mathematics and Mechanics / Zeitschrift für Angewandte Mathematik und Mechanik 98, 518-531.

Szeliga, D., Gawąd, J., Pietrzyk, M., 2004. Parameters identification of material models based on the inverse analysis. International Journal of Applied Mathematics and Computer Science 14, 549-556.

Taleb, L., Cailletaud, G., Saï, K., 2014. Experimental and numerical analysis about the cyclic behavior of the 304L and $316 \mathrm{~L}$ stainless steels at $350^{\circ} \mathrm{C}$. International Journal of Plasticity $61,32-48$.

Tanaka, E., 1994. A nonproportionality parameter and a cyclic viscoplastic constitutive model taking into account amplitude dependences and memory effects of isotropic hardening. European Journal of Mechanics A-solids 13, 155 $-173$.

Van Do, V.N., Lee, C.-H., Chang, K.-H., 2015. A constitutive model for uniaxial/multiaxial ratcheting behavior of a duplex stainless steel. Materials \& Design (1980-2015) 65, 1161-1171.

Voyiadjis, G.Z., Hoseini, S.H., Farrahi, G.H., 2012. A Plasticity Model for Metals With Dependency on All the Stress Invariants. Journal of Engineering Materials and Technology 135, 011002-011002-011013.

Xu, L., Nie, X., Fan, J., Tao, M., Ding, R., 2016. Cyclic hardening and softening behavior of the low yield point steel BLY160: Experimental response and constitutive modeling. International Journal of Plasticity 78, 44-63.

Yu, D., Chen, G., Yu, W., Li, D., Chen, X., 2012. Visco-plastic constitutive modeling on Ohno-Wang kinematic hardening rule for uniaxial ratcheting behavior of Z2CND18.12N steel. International Journal of Plasticity 28, 88-101. 
Zhang, J., Jiang, Y., 2008. Constitutive modeling of cyclic plasticity deformation of a pure polycrystalline copper. International Journal of Plasticity 24, 1890-1915.

Zhang, S.-L., Xuan, F.-Z., 2017. Interaction of cyclic softening and stress relaxation of 9-12\% Cr steel under straincontrolled fatigue-creep condition: Experimental and modeling. International Journal of Plasticity 98, 45-64.

Zhou, J., Sun, Z., Kanouté, P., Retraint, D., 2018. Experimental analysis and constitutive modelling of cyclic behaviour of $316 \mathrm{~L}$ steels including hardening/softening and strain range memory effect in LCF regime. International Journal of Plasticity 107, 54-78. 